\title{
Nanotized PPAR $\alpha$ Overexpression Targeted to Hypertrophied Myocardium Improves Cardiac Function by Attenuating the p53-GSK3 $\beta$-Mediated Mitochondrial Death Pathway
}

\author{
Santanu Rana, ${ }^{1}$ Ritwik Datta, ${ }^{1}$ Ratul Datta Chaudhuri, ${ }^{1}$ Emeli Chatterjee, \\ Mamta Chawla-Sarkar, and Sagartirtha Sarkar ${ }^{1}$
}

\begin{abstract}
Aims: Metabolic remodeling of cardiac muscles during pathological hypertrophy is characterized by downregulation of fatty acid oxidation (FAO) regulator, peroxisome proliferator-activated receptor alpha (PPAR $\alpha)$. Thereby, we hypothesized that a cardiac-specific induction of PPAR $\alpha$ might restore the FAO-related protein expression and resultant energy deficit. In the present study, consequences of PPAR $\alpha$ augmentation were evaluated for amelioration of chronic oxidative stress, myocyte apoptosis, and cardiac function during pathological cardiac hypertrophy.

Results: Nanotized PPAR $\alpha$ overexpression targeted to myocardium was done by a stearic acid-modified carboxymethyl-chitosan (CMC) conjugated to a 20-mer myocyte-targeted peptide (CMCP). Overexpression of $P P A R \alpha$ ameliorated pathological hypertrophy and improved cardiac function. Augmented PPAR $\alpha$ in hypertrophied myocytes revealed downregulated p53 acetylation (lys 382), leading to reduced apoptosis. Such cells showed increased binding of PPAR $\alpha$ with p53 that in turn reduced interaction of p53 with glycogen synthase kinase-3 $\beta$ (GSK3 $\beta$ ), which upregulated inactive phospho-GSK3 $\beta$ (serine [Ser]9) expression within mitochondrial protein fraction. Altogether, the altered molecular milieu in PPAR $\alpha$-overexpressed hypertrophy groups restored mitochondrial structure and function both in vitro and in vivo.

Innovation: Cardiomyocyte-targeted overexpression of a protein of interest (PPAR $\alpha$ ) by nanotized plasmid has been described for the first time in this study. Our data provide a novel insight towards regression of pathological hypertrophy by ameliorating mitochondrial oxidative stress in targeted $P P A R \alpha$-overexpressed myocardium.

Conclusion: PPAR $\alpha$-overexpression during pathological hypertrophy showed substantial betterment of mitochondrial structure and function, along with downregulated apoptosis. Myocardium-targeted overexpression of $P P A R \alpha$ during pathological cardiac hypertrophy led to an overall improvement of cardiac energy deficit and subsequent cardiac function, thereby, opening up a potential avenue for cardiac tissue engineering during hypertrophic cardiac pathophysiology. Antioxid. Redox Signal. 30, 713-732.
\end{abstract}

Keywords: cardiomyocyte, cardiac hypertrophy, $\operatorname{PPAR} \alpha$, apoptosis, mitochondria, targeted gene therapy

\section{Introduction}

$\mathbf{P}$ ATHOLOGICAL CARDIAC HYPERTROPHY at cellular level is manifested by increased myocyte size and reduced contractility (22). The pathophysiology involves activation of fetal genes [e.g., atrial natriuretic factor $(A N F)$ and $\beta$-myosin heavy chain $(\beta-M H C)]$ and convergence of various risk factors leading to heart failure $(8,61)$. The excess energy demand of contractile machinery in normal adult myocytes is met by an upregulated fatty acid metabolism via activation of key genes such as peroxisome proliferator-activated receptor alpha $(P P A R \alpha)$ and utilization by genes such as adipose

\footnotetext{
${ }^{1}$ Department of Zoology, University of Calcutta, Kolkata, India.

${ }^{2}$ Division of Virology, National Institute of Cholera and Enteric Diseases, Kolkata, India.
} 


\section{Innovation}

The study establishes welfare of fatty acid oxidation signaling preservation by targeted overexpression of nanotized peroxisome proliferator-activated receptor alpha $(P P A R \alpha)$ during cardiac hypertrophy and points toward the role of PPAR $\alpha$ in stabilization of myocyte mitochondria by moderating oxidative stress and resultant apoptotic load. Molecular exploration of such cells revealed that interaction of PPAR $\alpha$ with p53 C-terminal domain inhibits activation of glycogen synthase kinase- $3 \beta$ (GSK3 $\beta)$; this contributes to improved structural integrity and functional quality of myocyte mitochondria during hypertrophy. Thus, targeted overexpression of $P P A R \alpha$ arbitrates improvement of cardiac pathophysiology by resilience of myocyte mitochondria and precipitating further functional deterioration. Overall, the myocyte-targeted PPAR $\alpha$ augmentation strategy translates to a novel therapeutic regimen in molecular engineering of the diseased myocardium.

triglyceride lipase (ATGL) (27). Proteomic data from hypertrophied cardiac tissues have shown downregulated fatty acid oxidation (FAO) and a perinatal shift to glycolysis (46, 60) compared with the control group. However, recent preclinical studies have suggested that sustenance of FAO in cardiac hypertrophy may be instrumental toward preservation of myocardial energetics and function (27).

The hypertrophic stress responses in cardiomyocytes are known to upregulate and acetylate p53 (16). Acetylated p53 has been associated with mitochondrial apoptotic pathways (40) and, along with glycogen synthase kinase-3 $\beta$ (GSK3 $\beta$ ), plays a critical role in balancing pro- or antiapoptotic signals, integrating stress response to mitochondria for determination of cell fate (5). Concurrently, classical apoptotic signaling pathways lead to changes in mitochondrial structure and function (48). Basal (activated) state of GSK3 $\beta$ has been implicated in mitochondrial membrane depolarization and cytochrome-c release, whereas GSK3 $\beta$ (serine [Ser]9) phos- phorylation (inactivated) stabilizes mitochondria and provides myocyte protection $(30,52)$. Inhibition of p53 and GSK3 $\beta$ activation thus emerges as an important target for stabilization of mitochondrial integrity and apoptosis in myocytes during cardiac hypertrophy.

This study reports a myocyte-specific delivery system for encapsulated overexpression vectors by a stearic acid-modified carboxymethyl-chitosan (CMC) nanopolymeric vector conjugated to a 20-mer myocyte-targeted peptide (CMCP) (57). Our study shows for the first time that myocardium-specific overexpression of $P P A R \alpha$ during pathological hypertrophy binds the C-terminal domain of p53 leading to inactivation of p53 and GSK3 $\beta$. Mitochondria of such overexpressed cells showed structural and functional improvement along with reduced apoptotic load. The study discerns the molecular mechanism of restoration of cardiac energy deficit, regression of cardiac hypertrophy, and consequent improvement of cardiac function in ligated rat hearts due to targeted overexpression of $P P A R \alpha$.

\section{Results}

\section{$\operatorname{PPAR} \alpha$ modulation in cardiac hypertrophy}

Western blot analyses showed significant downregulation of PPAR $\alpha$ (in vitro: $2.73 \pm 0.025$-fold, $p<0.01$ and in vivo: $3.05 \pm 0.27$-fold, $p<0.01$ ) and ATGL (in vitro: $3.54 \pm 0.27$ fold and in vivo: $3.02 \pm 0.32$-fold, $p<0.001)$ during hypertrophy compared with respective control groups (Fig. 1A; Supplementary Fig. S1; Supplementary Data are available online at www.liebertpub.com/ars).

\section{Characterization of nanopolymeric delivery system}

Biophysical characterizations confirmed successful encapsulation of $P P A R \propto$ by CMCP and among the various encapsulated weight ratios, 1:50 (CMCP:pcDNA) showed significant retention and the encapsulation efficiency was $77.81 \%( \pm 4.8)$. $P P A R \alpha$ encapsulated within CMCP at 1:50 weight ratio showed a hydrodynamic diameter of $235 \mathrm{~nm}, \%$ polydispersity of 9.7 , and $\zeta$ potential of $+14.8 \mathrm{mV}$ (Fig. $1 \mathrm{~B}, \mathrm{C})$.

FIG. 1. Characterization of cardiac tissue-targeted overexpression of PPAR $\alpha$ during hypertrophy. (A) Western blot analyses showing downregulated expression of PPAR $\alpha$ and ATGL during cardiac hypertrophy compared with respective controls both in vitro and in vivo. GAPDH was used as loading control. $n=5$ for each group. (B) Gel retardation assay with different weight ratios of CMCP-encapsulated overexpression plasmids (CMCP:pcDNA) revealed successful condensation at a nanopolymer to plasmid weight ratio of 1:50. (C) Dynamic light scattering analysis for hydrodynamic diameter, \% polydispersity, and $\zeta$ potential of PPAR $\alpha$-encapsulated CMCP (1:50 weight ratio). (D) Western blot analyses by anti-his antibody showing in vitro overexpression of $P P A R \alpha$ by CMC and CMCP-mediated delivery. CMCP-mediated intravenous delivery of PPAR $\alpha$ overexpression vector in vivo showed cardiac tissue-targeted expression of PPAR $\alpha$-his compared with bystander organs, whereas CMC-mediated delivery showed no difference in expression of PPAR $\alpha$-his among the organs studied. $n=5$ for each group. (E) Immunofluorescence analysis showing successful expression of his-tag (green fluorescence) in myocytes transfected with CMCP-encapsulated PPAR $\alpha$ (i-v) compared with CMCP-encapsulated empty vectortransfected cells (vi-x). Myocytes were counterstained with $\alpha$-sarcomeric actin (red). Nuclei were stained with DAPI (blue). $n=5$ for each group. Bar $=20 \mu M, 100 \times$ magnification for all panels. (F) Immunofluorescence analysis of myocardial tissue sections showing successful expression of his-tag by CMCP-encapsulated PPAR $\alpha$ overexpression vector (i-iv) compared with empty vector-overexpressed myocardium (v-viii). Myocytes were counterstained with $\alpha$-sarcomeric actin (red). Nuclei were stained with DAPI (blue). $n=5$ for each group. Bar $=20 \mu M, 100 \times$ magnification for all panels. (G) Graph showing significant increase in percent "his-" positive cells within heart tissues by CMCP-encapsulated PPAR $\alpha$ overexpression compared with bystander organs, namely, the brain, liver, kidney, and lungs. $n=5$, for each group. ***p<0.001 with respect to bystander organs. ATGL, adipose triglyceride lipase; CMC, steric acid-modified carboxymethyl-chitosan; CMCP, CMC conjugated to a 20-mer myocyte-targeted peptide; DAPI, 4', 6-diamidino-2-phenylindole; GAPDH, glyceraldehyde 3phosphate dehydrogenase; His, histidine; PD, polydispersity; PPAR $\alpha$, peroxisome proliferator-activated receptor alpha. To see this illustration in color, the reader is referred to the web version of this article at www.liebertpub.com/ars 

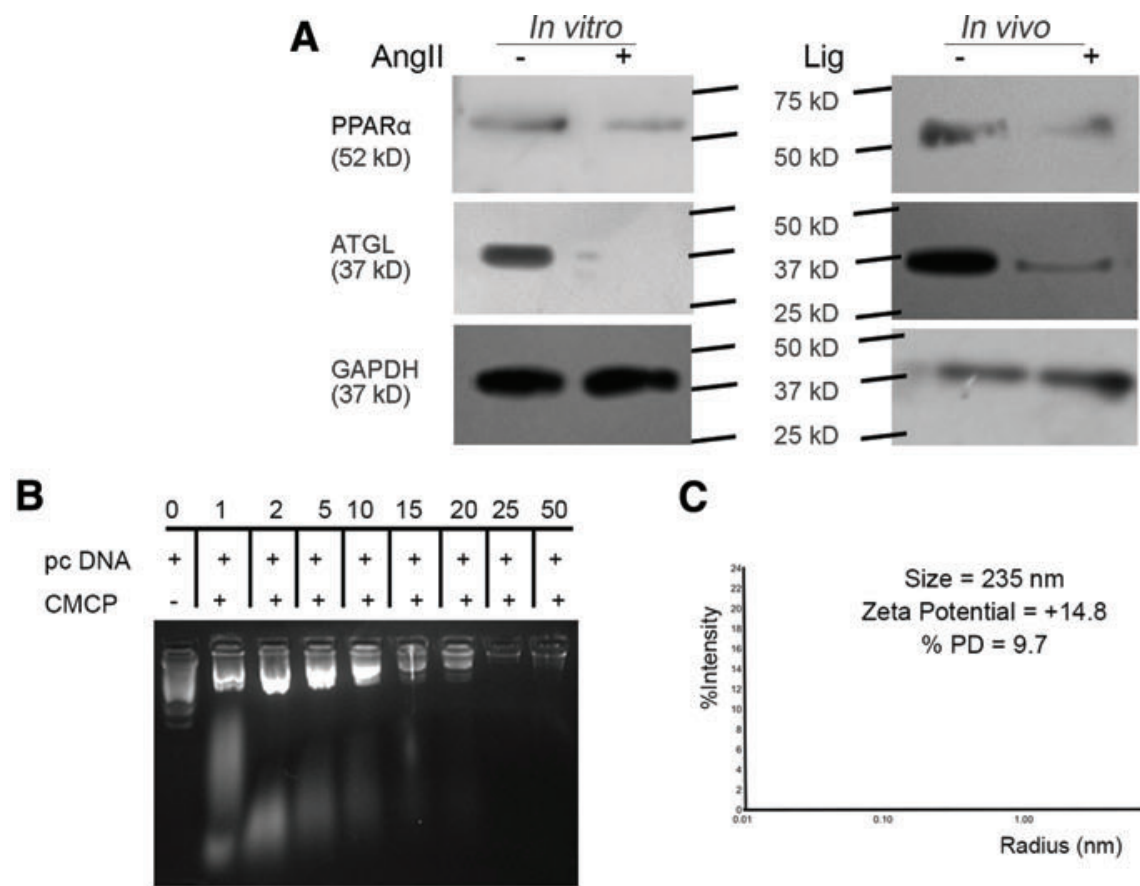

C
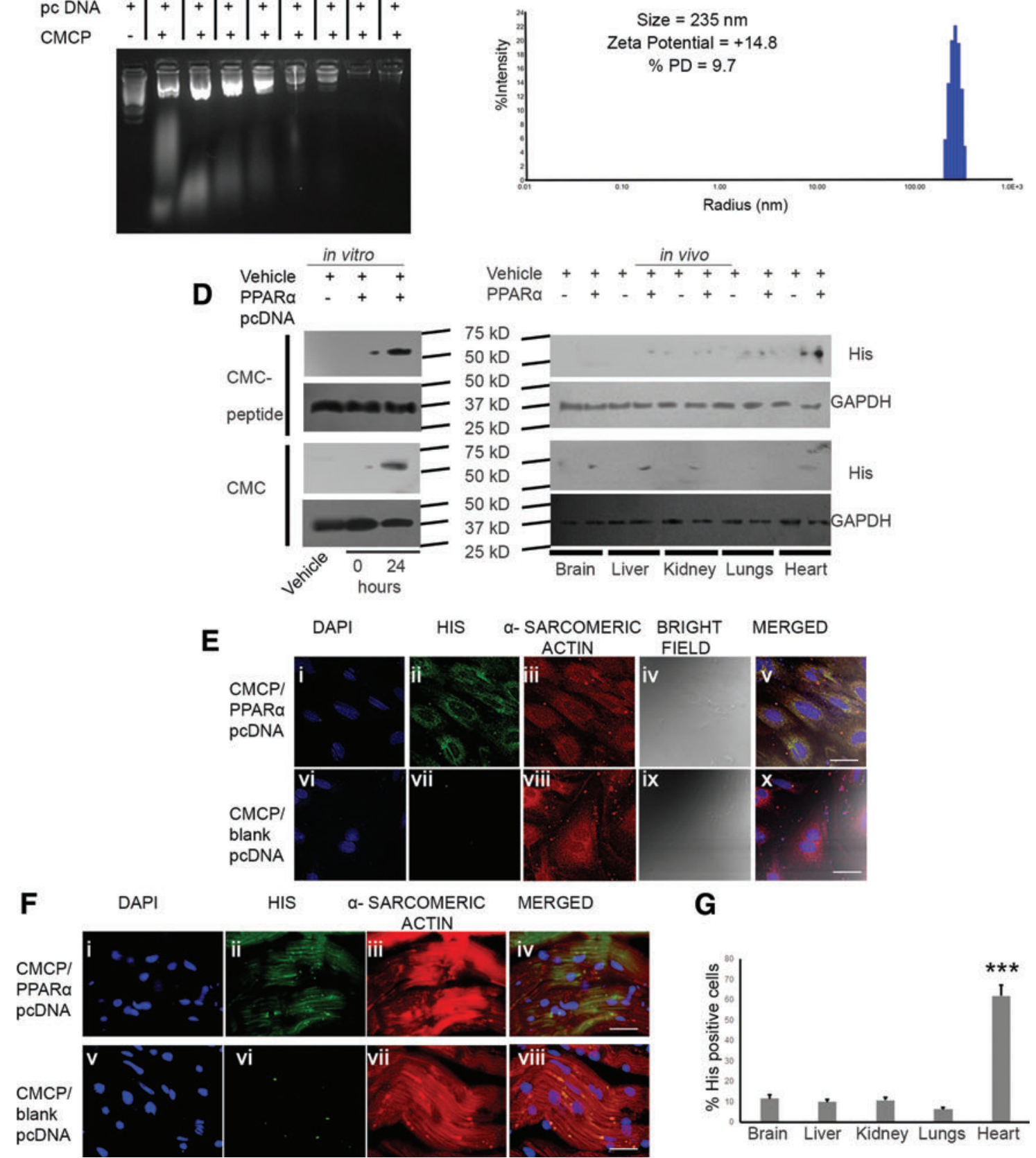
Scanning electron microscopic analysis of CMCP and CMCPencapsulated PPAR $\alpha$ showed homogenous size distribution of the nanopolymers (Supplementary Fig. S2). Moreover, serum incubation of CMCP-encapsulated PPAR $\alpha$ at different time periods from $30 \mathrm{~min}$ to $24 \mathrm{~h}$ revealed substantial integrity of the plasmid DNA within CMCP encapsulation, whereas free pcDNA on serum incubation showed degradation within $1 \mathrm{~h}$ (Supplementary Fig. S2).

\section{Myocyte-targeted overexpression of PPAR $\alpha$ by nanopolymer}

CMCP polyplex and CMC-mediated overexpression of $P P A R \propto$ in myocytes were estimated by Western blot analyses with anti-his antibody that showed plasmid transfection efficiency by CMCP is $1.91 \pm 0.25$-fold higher compared with $\mathrm{CMC}$ at $24 \mathrm{~h}$. Furthermore, rats overexpressed with $P P A R \alpha /$ CMCP showed significantly higher cardiac tissue-specific expression of histidine, "his," compared with other organs, namely, the brain $(5.42 \pm 0.35$-fold), liver $(6.3 \pm 0.24$ fold), kidney (5.77 \pm 0.42 -fold), and lungs $(9.92 \pm 0.69$ fold). CMC-mediated overexpression of PPAR $\alpha$ showed no significant difference in "his" expression among different organs studied (Fig. 1D; Supplementary Fig. S1). Confocal microscopy confirmed targeted transfection by "his" positivity in vitro and in vivo from myocytes counterstained with $\alpha$-sarcomeric actin. Moreover, CMCP-mediated overexpression revealed significantly higher percentage of "his-" positive myocytes in vivo the myocardium compared with other organs (Fig. 1E-G).

Overexpression of PPAR $\alpha$ by CMCP encapsulation in ligated samples showed significant restoration of PGC1 $\alpha$ (4.21 \pm 0.36 -fold), ATGL (4.9 \pm 0.34 -fold), and CPT1 $\alpha$
(2.49 \pm 0.16 -fold) compared with nanotized empty plasmidtargeted ligated groups (Supplementary Fig. S3 and S4).

\section{Regression of cardiac hypertrophy via targeted overexpression of PPAR $\alpha$}

Overexpression of $P P A R \alpha$ in angiotensin II (AngII)treated hypertrophied myocytes showed significant downregulation of the hypertrophy marker gene expression in vitro $A N F(2.14 \pm 0.28$-fold) and $\beta$ - MHC (2.35 \pm 0.26 -fold) when compared with empty plasmid-transfected hypertrophied myocytes. Targeted overexpression of nanotized PPAR $\alpha$ in ligated rat hearts also revealed significantly downregulated $A N F$ (4.56 \pm 0.21 -fold) and $\beta-M H C$ (4.34 \pm 0.37-fold; Supplementary Fig. 2A) when compared with nanotized empty plasmid-targeted hypertrophied rat hearts.

Overexpression of nanotized $P P A R \alpha$ in ligated rats resulted in significant reduction of myocyte cross-sectional area $\left(518.4 \pm 19.2 \mu \mathrm{m}^{2}\right)$ as well as heart weight $(\mathrm{HW})$ to body weight $(\mathrm{BW})$ ratio $(3.32 \pm 0.16)$ compared with nanotized empty plasmid-targeted ligated rats $\left(678.6 \pm 39.1 \mu \mathrm{m}^{2}\right.$ and 4.57 \pm 0.09 , respectively) (Fig. 2B, C; Supplementary Fig. S5).

Myocyte-targeted overexpression of nanotized $P P A R \alpha$ also showed improved cardiac function, as evidenced by a decreased left ventricular diastolic dimension (LVDd: $5.25 \pm 0.32 \mathrm{~mm}$ ), and restored the $\%$ fractional shortening (\%FS: $50.74 \pm 3.72 \%$ ) during ligation compared with nanotized empty plasmid-targeted ligated rats (LVDd: $7.62 \pm 0.53 \mathrm{~mm}$; $\%$ FS: $38.16 \pm 4.06 \%$ ), as revealed by M-mode echocardiographic analyses (Fig. 2D). Other echocardiographic parameters, namely, left ventricular systolic internal dimensions (LVDs), left ventricular anterior wall diameter in diastole

FIG. 2. Overexpression of PPAR $\alpha$ mediates regression of hypertrophy and amelioration of apoptosis during pathological cardiac hypertrophy. (A) RT-PCR analyses showing significantly reduced expression of $A N F$ and $\beta-M H C$ in CMCPencapsulated $P P A R \alpha$-overexpressed hypertrophy groups compared with CMCP-encapsulated empty vector-overexpressed hypertrophy groups both in vitro and in vivo. GAPDH was used as loading control. Corresponding graphs show relative fold changes in gene expression from different experimental groups. $n=5$, for each group. *** $p<0.001$ with respect to control groups; ${ }^{\dagger \dagger} p<0.01$ with respect to hypertrophy groups. (B) H\&E staining of cardiac tissues revealed regression of myocyte CSA $\left(\mu \mathrm{m}^{2}\right)$ during myocyte-targeted overexpression of $P P A R \alpha$ in ligated rat heart $\left(518.4 \pm 19.2 \mu \mathrm{m}^{2}\right.$; iii) compared to ligated group $\left(678.6 \pm 39.1 \mu \mathrm{m}^{2}\right.$; ii). Corresponding graph showing changes in myocyte-CSA among different groups. (Scale Bar $=100 \mu \mathrm{m}$, magnification $=60 \times$ ). Sham operated (i) and ligated animals were treated with CMCP encapsulated empty vector. $n=5$, for each group. $* * * p<0.001$ with respect to sham treated control groups; ${ }^{\dagger \dagger} p<0.01$ with respect to ligated rats. (C) Graphs showing significant restoration of HW/BW ratios $(\mathrm{mg} / \mathrm{g})$ during cardiac-specific overexpression of $P P A R \alpha(3.32 \pm 0.16)$ in ligated group compared with ligated rat hearts $(4.57 \pm 0.09)$. Sham operated and ligated animals were treated with CMCP-encapsulated empty vector. $n=5$ for each group. $*_{* *}^{*} p<0.001$ with respect to sham treated control groups; ${ }^{\dagger \dagger} p<0.01$ with respect to ligated rats. (D) Graphs showing restoration of \%FS and decreased LVDd during cardiac-specific overexpression of $P P A R \alpha$ in ligated group compared with ligated rat hearts. Sham operated and ligated animals were treated with CMCP-encapsulated empty vector. $n=5$, for each group. $* * * p<0.001$ with respect to sham treated control groups; ${ }^{\dagger \dagger} p<0.01$ with respect to ligated rats. (E) Western blot analyses showing significantly downregulated caspase-3 cleavage and mitochondrial cytochrome-C release in $P P A R \alpha-$ overexpressed hypertrophy groups compared with empty vector-targeted hypertrophy groups both in vitro and in vivo. GAPDH was used as an internal cytosolic and total protein loading control and COX IV was used as mitochondrial loading control. $n=5$ for each group. For in vitro panel: lanes $\mathrm{C}$, control myocytes treated with empty plasmid; A, AngII-treated myocytes with empty plasmid and $\mathrm{A}+\mathrm{P}, P P A R \alpha$-overexpressed hypertrophied myocytes and; and for in vivo panel: lanes $\mathrm{S}$, sham operated control treated with empty plasmid; L, ligated rat heart treated with empty plasmid and L+P, myocyte-targeted $P P A R \alpha$-overexpressed ligated heart. (F) Caspase-3 activity assay showing significant reduction of caspase-3 activity in ligated rat hearts overexpressed with $P P A R \alpha$ compared with hypertrophy group. Sham operated and ligated animals were treated with CMCP-encapsulated empty vector. $n=5$ for each group. $*^{* *} p<0.001$ with respect to sham treated control groups; ${ }^{\dagger \dagger} p<0.01$ with respect to hypertrophy groups. $\beta$-MHC, $\beta$-myosin heavy chain; \%FS, \% fractional shortening; ANF, atrial natriuretic factor; AngII, angiotensin II; BW, body weight; COX IV, cytochrome c oxidase subunit IV; CSA, cross-sectional area; H\&E, hematoxylin and eosin; HW, heart weight; LVDd, left ventricular diastolic dimension; PCR, polymerase chain reaction; RT, reverse transcription. To see this illustration in color, the reader is referred to the web version of this article at www.liebertpub.com/ars 
A

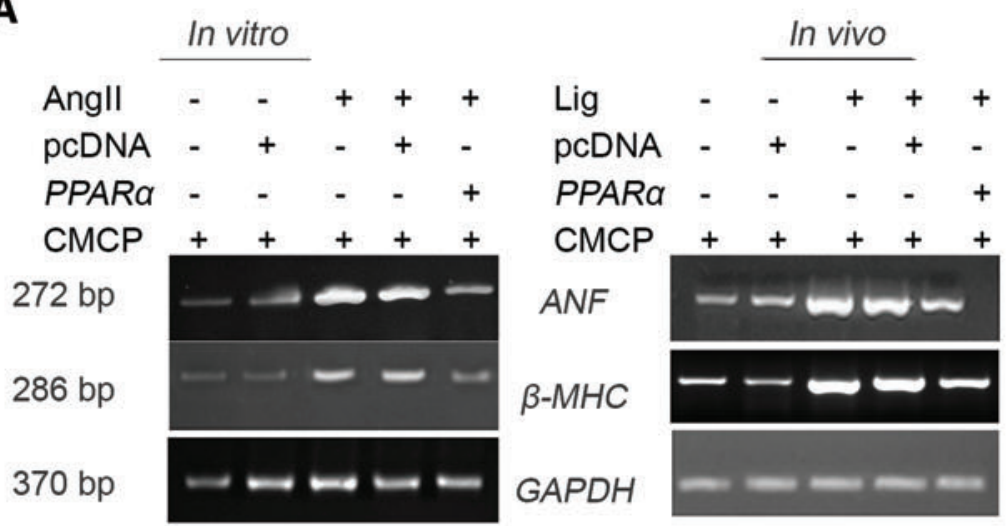

B

Sham+empty vector

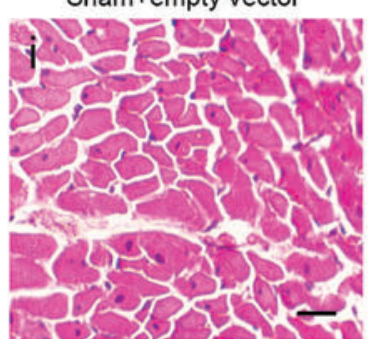

Ligated+empty vector

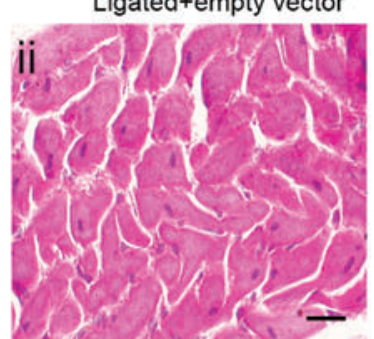

Ligated + PPARa
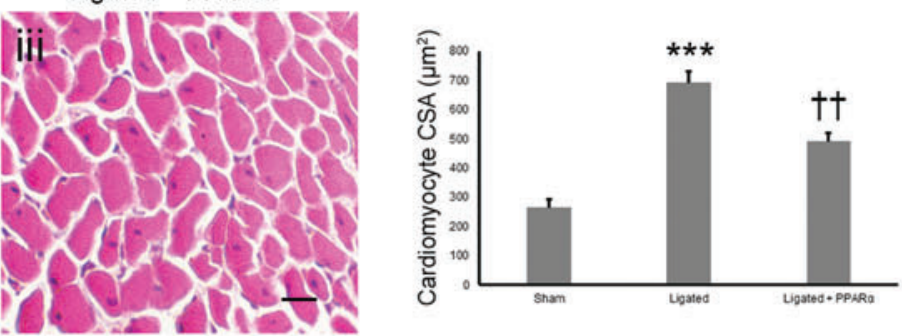

C

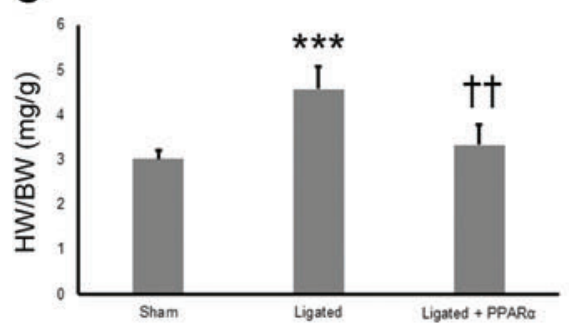

D
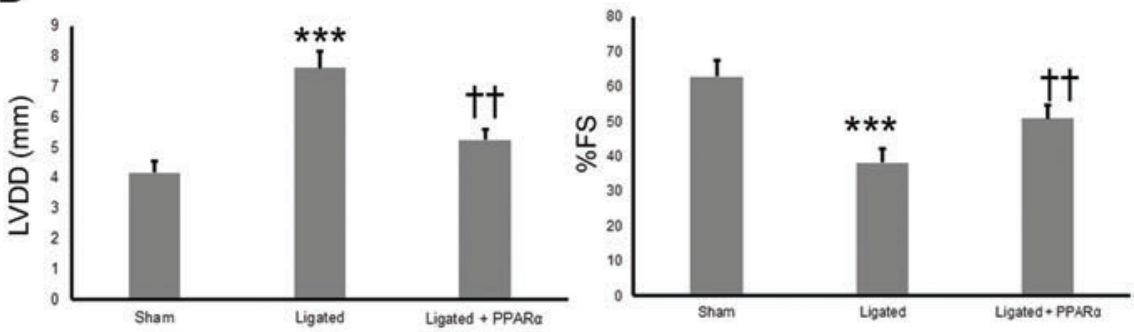

E

Caspase 3

(35 kD \& 19

$\mathrm{kD})$

GAPDH

(37 kD)

CytC (cyto)

(14 kD)

CytC (mito)

(14 kD)

COXIV

(17 kD)

GAPDH

(cyto)

(37 kD)
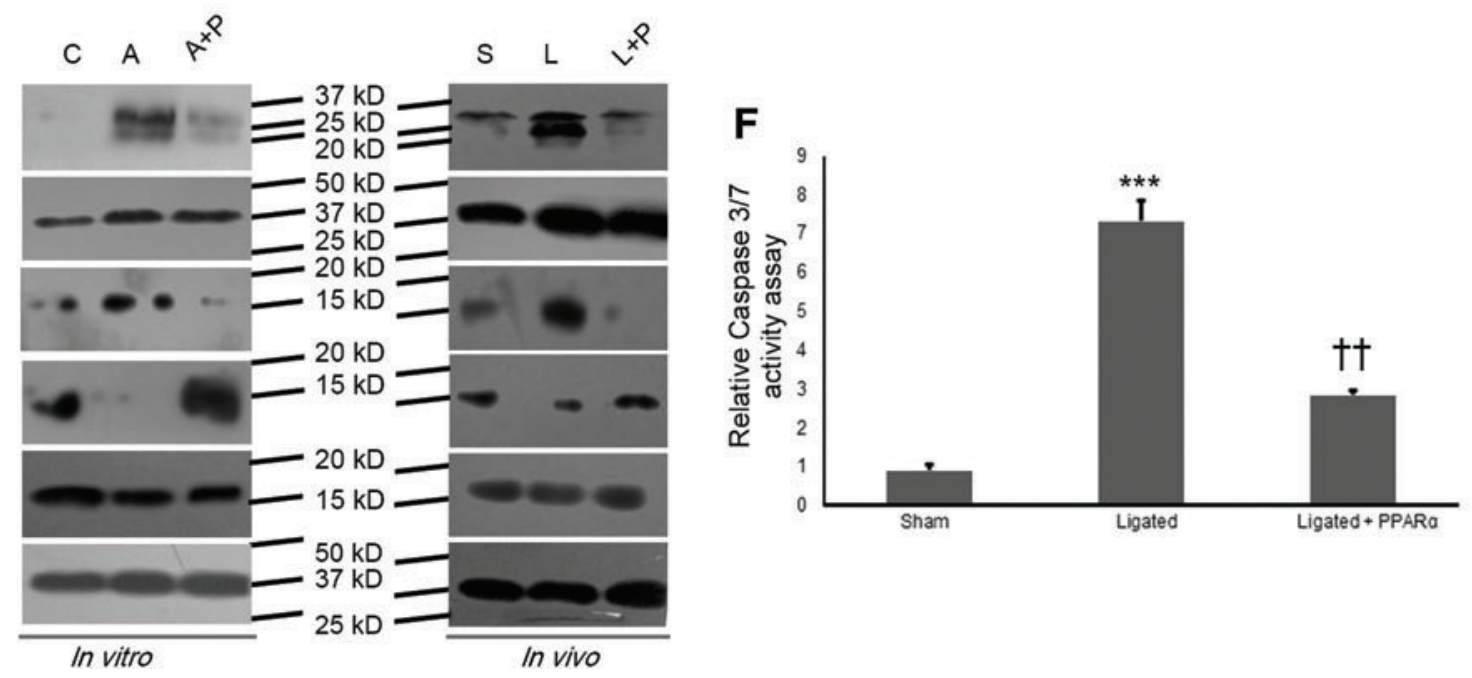

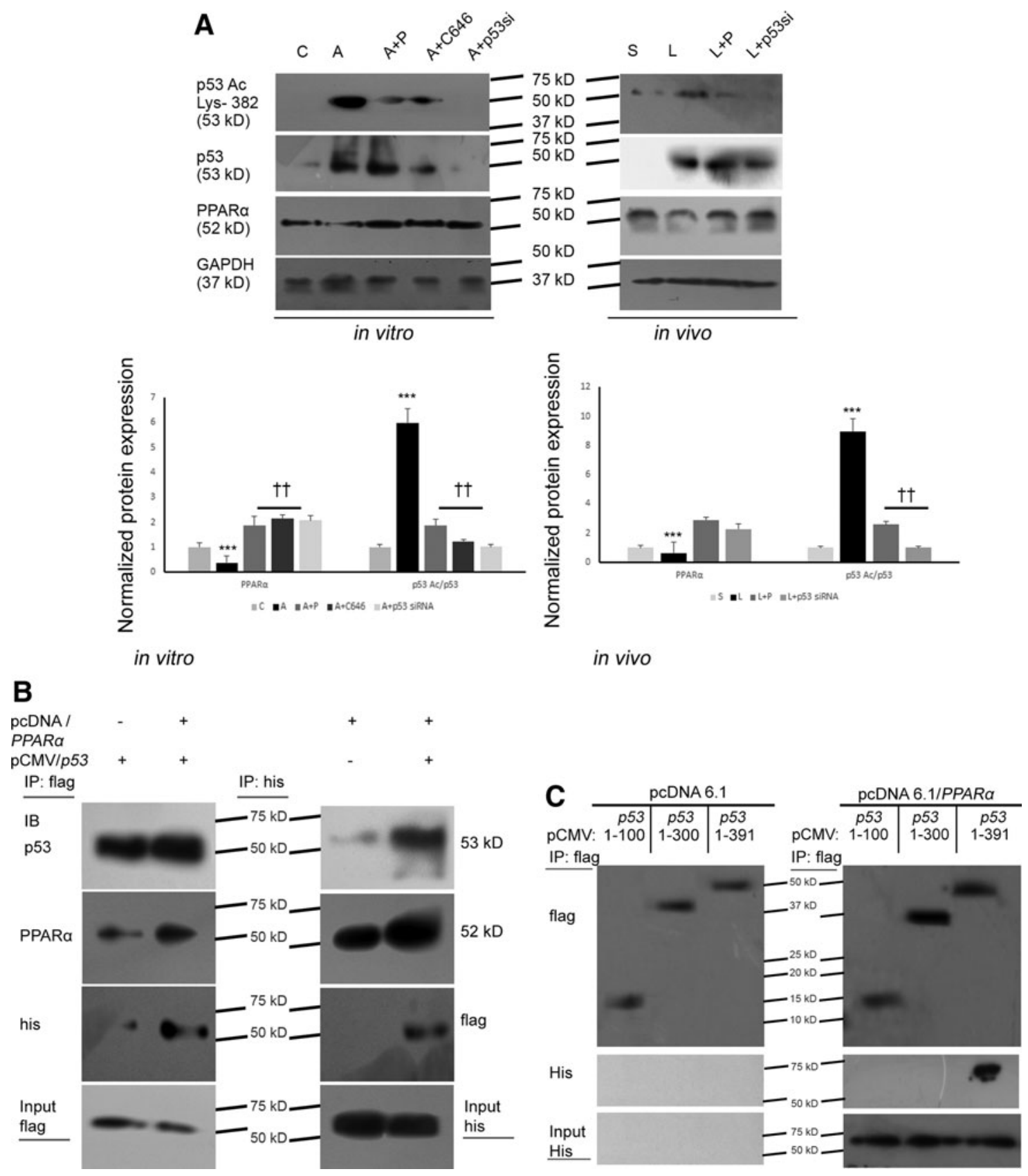

FIG. 3. Overexpression of PPAR $\alpha$ during hypertrophy attenuates p53 acetylation. (A) Western blot analyses showing changes in expression of acetylated p53 acetylation (lys 382) to total p53 and PPAR $\alpha$ expression among different treatment groups both in vitro and in vivo. For in vitro: lanes C, control myocytes; A, AngII-treated myocytes; A+P, $P P A R \alpha-$ overexpressed hypertrophied myocytes; A+C646, C646-treated hypertrophied myocytes and A+p53si, p53 siRNA-treated hypertrophied myocytes; and for in vivo: lanes S, sham operated control heart; L, ligated rat heart; L+P, myocyte-targeted $P P A R \alpha$-overexpressed ligated heart and $\mathrm{L}+\mathrm{p} 53 \mathrm{si}$, myocyte-targeted p53 siRNA-treated ligated heart. Control and hypertrophy groups were treated with DMSO or NS siRNA or empty plasmid, yielding similar results. $n=5$ for each group. Corresponding graphical representation showing expression pattern of the proteins from the different experimental groups; all relative protein expressions were $\log _{2}$ transformed. ${ }^{* * *} p<0.001$ with respect to control groups; ${ }^{\dagger \dagger} p<0.01$ with respect to hypertrophy groups. (B) Coimmunoprecipitation analyses using anti-flag antibody followed by immunoblotting with anti-his antibody revealed significant binding between p53-flag and PPAR $\alpha$-his in cotransfected myocytes compared with cells transfected with $p 53 /$ pCMV alone. Reverse coimmunoprecipitation using anti-his antibody followed by immunoblotting with anti-flag antibody showed similar results. $n=5$ for each group. (C) Myocytes were cotransfected with $P P A R \alpha$ cloned in pcDNA-his and different domains of p53 cloned in CMV-flag. Coimmunoprecipitation analysis using anti-flag antibody followed by immunoblotting with anti-his antibody showed no interaction of PPAR $\alpha$ with the 1-100 aa and 1-300 aa domains of p53; however, substantial interaction was revealed with full-length p53 (1-391 aa regions). Transfection with empty vector was used as a negative control. $n=5$ for each group. aa, amino acid; CMV, cytomegalovirus immediate-early; DMSO, dimethyl sulfoxide; NS siRNA, non-specific siRNA. 
(LvAWDd), left ventricular anterior wall diameter in systole (LvAWDs), left ventricular posterior wall diameter in diastole (LvPWDd), and left ventricular posterior wall diameter in systole (LvPWDs), also support the previous findings (Supplementary Fig. S3B; Supplementary Table S1).
Overexpression of PPAR $\alpha$ downregulates

mitochondria-mediated apoptosis during hypertrophy

Nanotized PPAR $\alpha$-overexpressed hypertrophy samples showed reduced caspase- 3 cleavage both in vitro and in vivo along with downregulated caspase-3 activity (in vivo:
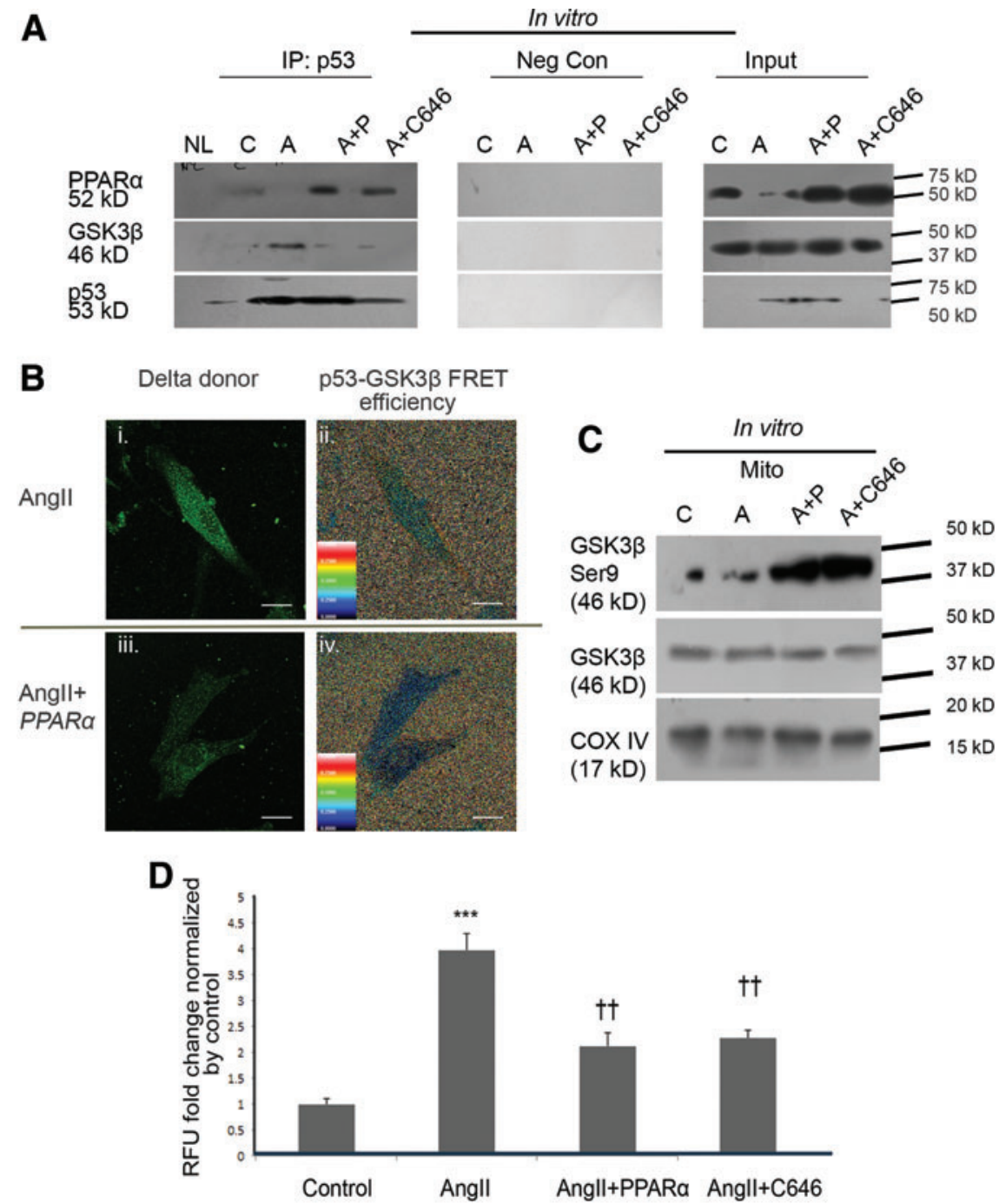

FIG. 4. Targeted overexpression of PPAR $\alpha$ in hypertrophy samples showed inhibition of p53-GSK3 $\beta$ interaction. (A) Coimmunoprecipitation analyses using anti-p53 antibody followed by immunoblotting with anti-PPAR $\alpha$ and anti-GSK3 $\beta$ antibodies in different experimental groups. $n=6$, for each group. Panel: lanes NL, no lysate; C, control myocytes; A, AngII-treated myocytes; A+P, PPAR $\alpha$-overexpressed hypertrophied myocytes and A+C646, C646-treated hypertrophied myocytes. C and A groups were treated with either DMSO or empty plasmid, yielding similar results. (B) Hypertrophied myocytes were immunostained for GSK3 $\beta$-TRITC (red) and p53-FITC (green); TRITC was subjected to 50\% photobleaching. FRAP showing significantly lower FRET efficiency of GSK3 $\beta$ with p53 in PPAR $\alpha$-overexpressed hypertrophied myocytes (iv) compared with empty vector-transfected hypertrophied cells (ii). Scale bar $=20 \mu M, 100 \times$ magnification for all panels. $n=3$ for each group. (i, iii) Delta donor (delta donor: postbleaching-prebleaching donor intensities); (ii, iv) FRET efficiency. (C) Western blot analyses from mitochondrial protein lysates showing upregulated GSK3 $\beta$ (Ser9) in PPAR $\alpha$-overexpressed hypertrophied myocytes compared with hypertrophied cells. COX IV was used as mitochondrial loading control. $n=6$ for each group. Panel: lanes C, control myocytes; A, AngII-treated myocytes; A+P, PPAR $\alpha$-overexpressed hypertrophied myocytes and A+C646, C646-treated hypertrophied myocytes. C and A groups were treated with either DMSO or empty plasmid, yielding similar results. (D) ROS assay showing significant reduction of oxidative stress in AngII-treated myocytes overexpressed with PPAR $\alpha$ compared with untreated hypertrophied groups. Hypertrophied myocytes treated with C646 were used as a positive control. C and A groups were treated with either DMSO or empty plasmid, yielding similar results. $n=5$ for each group. $* * * p<0.001$ with respect to control groups; ${ }^{\dagger} p<0.01$ with respect to hypertrophy groups. FRAP, fluorescence recovery after photobleaching; FRET, fluorescence resonance energy transfer; GSK $3 \beta$, glycogen synthase kinase- $3 \beta$; ROS, reactive oxygen species; Ser, serine. To see this illustration in color, the reader is referred to the web version of this article at www.liebertpub.com/ars 
$2.57 \pm 0.18$-fold) compared with nanotized empty plasmidtargeted hypertrophy group (Fig. 2E, F). Targeted overexpression of $P P A R \alpha$ in hypertrophy samples also showed significant downregulation of mitochondrial cytochrome-C release (in vitro: $2.67 \pm 0.15$-fold, $p<0.01$ and in vivo: $3.79 \pm$ 0.39 -fold, $p<0.01)$ compared with respective hypertrophy groups (Fig. 2E; Supplementary Fig. S6B).

\section{Overexpression of PPAR $\alpha$ inhibits $p 53$ acetylation during hypertrophy}

Nanotized $P P A R \alpha$-overexpressed hypertrophy groups revealed significantly downregulated p53 acetylation (lys 382) (in vitro: $2.18 \pm 0.19$-fold, $p<0.01$ and in vivo: $1.86 \pm 0.22$ fold, $p<0.01)$ compared with empty plasmid-treated hypertrophy groups (Fig. 3A). C646 treatment showed significant inhibition of p53 acetylation in vitro. Successful knockdown of p53 during hypertrophy by $p 53$ siRNA treatment both in vitro and in vivo was used as negative control. C646mediated inhibition of acetylated p53 or p53 knockdown by siRNA treatment in hypertrophy samples showed restoration of PPAR $\alpha$ expression compared with hypertrophy groups (Fig. 3A; Supplementary Fig. S7).

\section{$\operatorname{PPAR} \alpha$ binds $C$-terminal domain of p53}

Myocytes were transfected with p53-cytomegalovirus immediate-early (CMV) (flag tag) either in the presence or absence of $P P A R \alpha$-pcDNA (his-tag). Coimmunoprecipitation analyses, using the anti-flag antibody followed by Western blotting with anti-his antibody, showed substantial interaction between p53 and PPAR $\alpha$ in myocytes (Fig. 3B; Supplementary Fig. S7). Reverse coimmunoprecipitation yielded similar results. Furthermore, interactions between the two proteins were mapped to the domains of p53. Myocytes were cotransfected with PPAR $\alpha$-pcDNA (his-tag) and the p53 domains 1-100, 1-300, and 1-391 (full length) cloned in CMV (flag tag). Flag tag pull down followed by immunoblotting with anti-his antibody revealed considerable interaction of 1391 amino acid (aa) region of p53 with PPAR $\alpha$. However, the study did not reveal any substantial interaction of PPAR $\alpha$ with 1-100 and 1-300 aa regions of p53, suggesting that C-terminal domain (300-391 aa) of p53 might be critical for physical interaction with PPAR $\alpha$ (Fig. 3C; Supplementary Fig. S7).

\section{Overexpression of PPAR $\alpha$ modulates p53 and GSK3 $\beta$ binding during hypertrophy}

$P P A R \alpha$-overexpressed hypertrophied myocytes were found to be associated with increased binding of p53 with $\operatorname{PPAR} \alpha$ compared with hypertrophy group treated with empty vector. However, interaction of p53 with GSK3 $\beta$ was significantly reduced $(2.43 \pm 0.19$-fold, $p<0.01)$ in such $P P A R \alpha$-overexpressed hypertrophied cells compared with hypertrophy alone, as revealed by coimmunoprecipitation analyses. Similarly, reduced interaction of p53 with GSK3 $\beta$ was also found in C646-treated hypertrophied myocytes (3.31 \pm 0.34 -fold, $p<0.01)$ compared with hypertrophied myocytes (Fig. 4A; Supplementary Fig. S8A).

Comparative binding efficiency between p53 and GSK3 $\beta$ assessed by fluorescence recovery after photobleaching (FRAP) analyses showed a significantly lower fluorescence resonance energy transfer (FRET) efficiency (2.87 \pm 0.42 -fold, $p<0.01)$ in $P P A R \alpha$-overexpressed hypertrophied myocytes compared with empty vector-transfected hypertrophied cells, indicating reduced binding between $\mathrm{p} 53$ and GSK $3 \beta$ by increased PPAR $\alpha$-p53 interaction in hypertrophied myocytes (Fig. 4B).

Mitochondrial protein lysate from $P P A R \alpha$-overexpressed hypertrophied myocytes showed upregulated GSK3 $\beta$ (Ser9) expression levels $(3.26 \pm 0.34$-fold, $p<0.01)$ compared with empty vector-transfected hypertrophied cells (Fig. 4C; Supplementary Fig. S8B).

FIG. 5. Overexpression of PPAR $\alpha$ ameliorates mitochondrial oxidative stress during hypertrophy. (A) Immunofluorescence study using MitoSOX ${ }^{\mathrm{TM}}$ Red showing restoration of mitochondrial superoxide radical in $P P A R \alpha$-overexpressed hypertrophied myocytes (vii-ix) compared with hypertrophied cells (iv-vi). DAPI (blue fluorescence) was used to stain the nuclei. Control (i-iii) and hypertrophied cells were treated with empty plasmid. $n=5$ for each group. Scale bar $=20 \mu M, 100 \times$ magnification for all panels. (B) Immunofluorescence analyses showing changes in mitochondrial membrane potential as revealed by fluorescence intensity of aggregate (red fluorescence) to monomeric (green fluorescence) ratio of JC-1 stain. Such ratio was decreased in hypertrophied myocytes (v-viii) compared with control cells (i-iv), which was restored in PPAR $\alpha$-overexpressed hypertrophied cells (ix-xii). Hypertrophied myocytes treated with C646 (xiii-xvi) showed similar restoration. Nuclei were stained by DAPI (blue fluorescence). Control and hypertrophied myocytes were treated with either DMSO or empty plasmid, yielding similar results. $n=5$ for each group. Scale bar $=20 \mu M, 100 \times$ magnification for all panels. (C) Western blot analyses showing expressions of Drp1, Bax, Bid, and Tfam in mitochondrial protein lysates from different treatment groups both in vitro and in vivo. COX IV was used as mitochondrial loading control. $n=5$ for each group. For in vitro: lanes C, control myocytes; A, AngII-treated myocytes and A+P, $P P A R \alpha-$ overexpressed hypertrophied myocytes; and for in vivo: lanes S, sham operated control heart; L, ligated rat heart; and L+P, myocytetargeted PPAR $\alpha$-overexpressed ligated heart. Control and hypertrophy groups were treated with empty plasmid. Corresponding graphs showing relative changes in these protein expressions from different experimental groups both in vitro and in vivo. All relative protein expression values were $\log _{2}$ transformed. $* * * p<0.001$ with respect to control groups; ${ }^{\dagger} p<0.01$ with respect to hypertrophy groups. (D) Immunofluorescence study showing increased Tfam (red fluorescence) and decreased caspase-3 cleaved positive nuclei (green fluorescence) in PPAR $\alpha$-overexpressed hypertrophied myocytes (ix-xii) compared with hypertrophied cells (v-viii). DAPI (blue fluorescence) was used to stain the nuclei. Control (i-iv) and hypertrophied cells were treated with empty plasmid. $n=5$ for each group. Scale $\mathrm{bar}=20 \mu M, 100 \times$ magnification for all panels. Relative intensities of Tfam were calculated with respect to control samples, and caspase-3-positive nuclei were scored in different experimental groups. Corresponding graph showing caspase-3 nuclear localization is negatively associated with Tfam expression. Tfam expression values were $\log _{2}$ transformed. $* * * p<0.001$ with respect to control groups; ${ }^{\dagger} p<0.01$ with respect to hypertrophy groups. Bax, BCL2-associated X protein; Bid, BH3interacting domain death agonist; Drp1, dynamin-related protein 1; JC-1, 5, 5', 6, 6'-tetrachloro-1, 1', 3, 3'-tetraethylbenzimidazolylcarbocyanine iodide; Tfam, transcription factor of activated mitochondria. To see this illustration in color, the reader is referred to the web version of this article at www.liebertpub.com/ars 
PPAR $\alpha$ moderates mitochondrial oxidative stress and associated protein expression during hypertrophy

PPAR $\alpha$-overexpressed hypertrophied myocytes also showed significant regression of cellular reactive oxygen

A

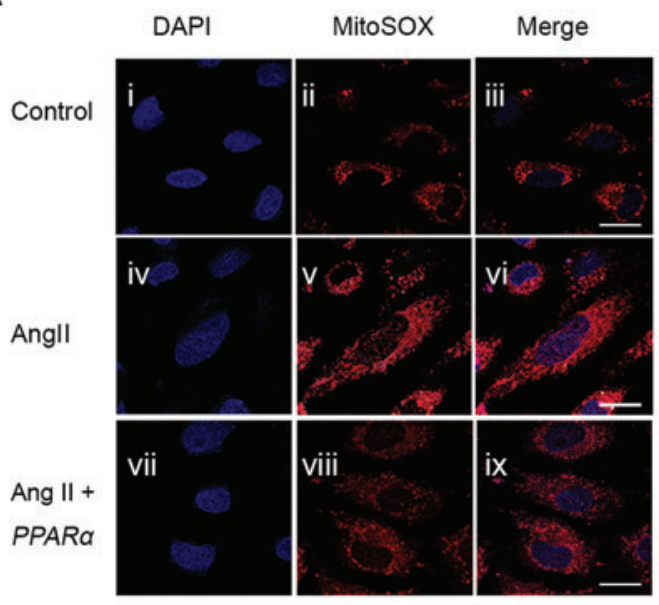

C

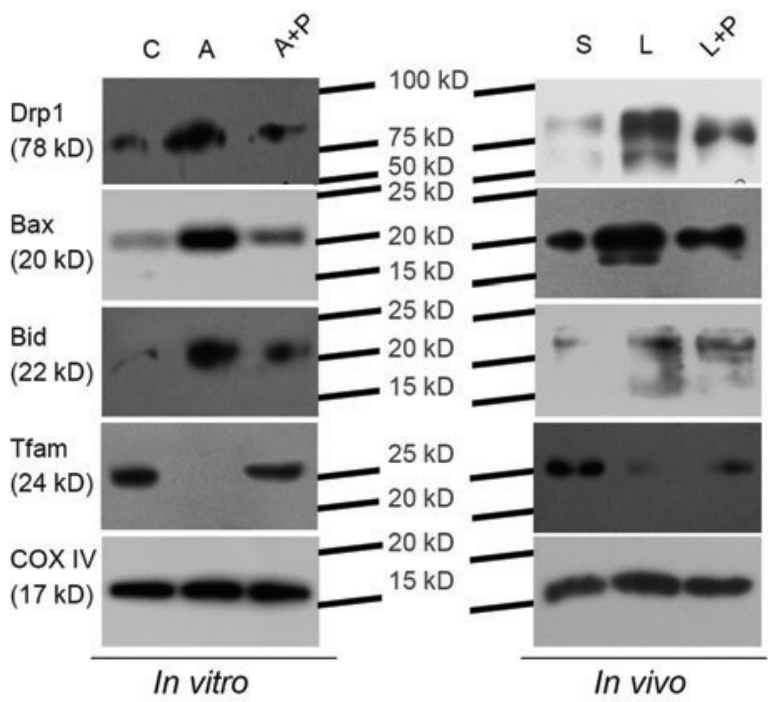

D

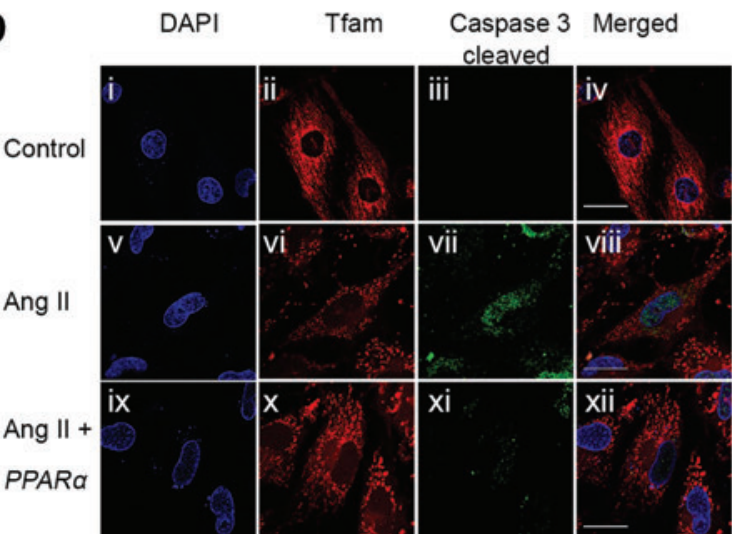

species (ROS) level (3.27 \pm 0.42 -fold) compared with hypertrophied cells (Fig. 4D). Moreover, substantially upregulated mitochondrial superoxide $\left(\mathrm{O}_{2}^{-}\right)$in hypertrophied myocytes compared with control was significantly restored by overexpression of $P P A R \alpha$, as revealed by MitoSOX ${ }^{\mathrm{TM}}$ Red

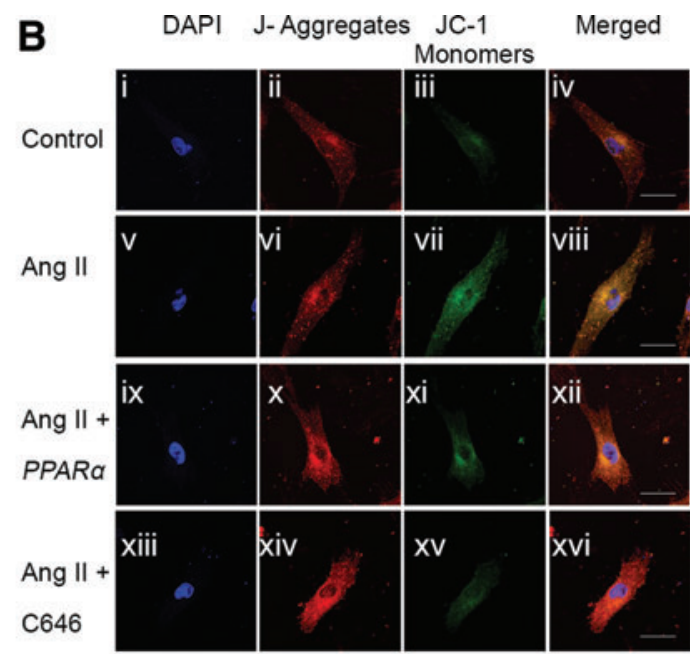

in vitro
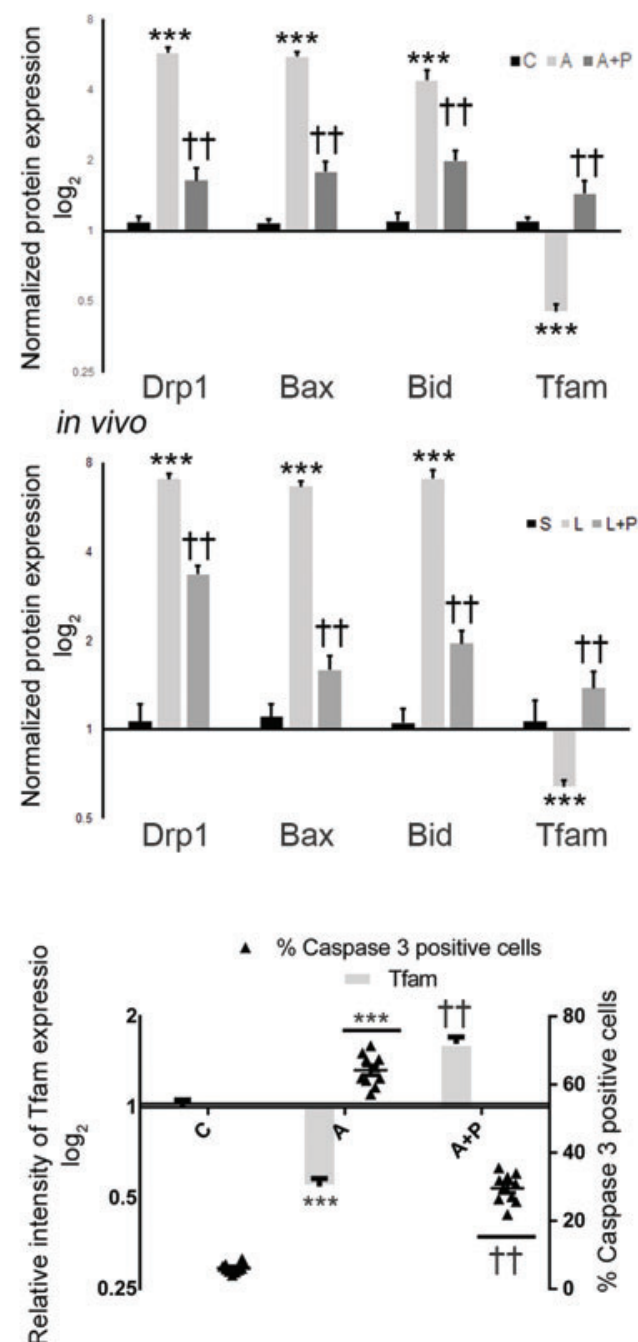
(Fig. 5A). Ratiometric analyses of 5, 5' , 6, 6'-tetrachloro-1, 1', 3, 3'-tetraethylbenzimidazolylcarbocyanine iodide (JC-1) aggregate to JC-1 monomer fluorescence intensity revealed substantially lower mitochondrial membrane potential $(\Delta \Psi \mathrm{m})$ in hypertrophied myocytes compared with control cells that was restored by overexpression of $P P A R \alpha$ in hypertrophied myocytes; similar results were observed by C646 treatment of hypertrophied myocytes (Fig. 5B; Supplementary Fig. S9).

Mitochondrial protein lysates of $P P A R \alpha$-overexpressed hypertrophy samples revealed significantly downregulated expression of dynamin-related protein 1 (Drp1; in vitro: $3.5 \pm$ 0.25-fold and in vivo: $2.09 \pm 0.15$-fold), BCL2-associated X protein (Bax; in vitro: $3.1 \pm 0.21$-fold and in vivo: $4.18 \pm 0.29$ fold), and BH3-interacting domain death agonist (Bid; in vitro: $2.19 \pm 0.19$-fold and in vivo: $3.61 \pm 0.28$-fold) compared with empty vector-treated hypertrophy group. Mitochondrial transcription factor of activated mitochondria (Tfam) expression showed significant downregulation in hypertrophy samples compared with control (in vitro: $2.43 \pm 0.2$-fold and in vivo: $1.65 \pm 0.11$-fold), which was again restored significantly by overexpression of $P P A R \alpha$ during hypertrophy (in vitro: $3.17 \pm 0.23$-fold and in vivo: $2.15 \pm$ 0.19-fold, respectively; Fig. 5C; Supplementary Fig. S10).

An inverse association was revealed between mitochondrial Tfam expression and nuclear cleaved caspase-3. PPAR $\alpha$ overexpressed hypertrophied myocytes showed a significantly reduced number of caspase-3-positive nuclei (2.16 \pm 0.23 -fold) and increased mitochondrial biogenesis (upregulated Tfam expression by $2.96 \pm 0.18$-fold) compared with hypertrophied cells (Fig. 5D).

\section{PPAR $\alpha$ improves myocyte mitochondrial number and structure during hypertrophy}

The relative amount of mitochondrial DNA (mtDNA) copy number was significantly downregulated in vitro $(4.12 \pm 0.26$-fold, $p<0.001)$ and in vivo $(4.67 \pm 0.34$-fold, $p<0.001)$ in empty plasmid-targeted hypertrophy samples compared with respective control groups and was found to be reversed in $P P A R \alpha$-overexpressed hypertrophy groups (in vitro: $2.46 \pm 0.32$-fold, $p<0.01$ and in vivo: $3.27 \pm 0.31$ - fold, $p<0.01)$. Similar results were also observed either by C646 or $p 53$ siRNA treatment during hypertrophy (Fig. 6A; Supplementary Fig. S11).

MitoTracker ${ }^{\circledR}$ Red-loaded control myocytes showed two different morphological structures of mitochondria, namely, reticulate or tubular and punctate networks (Fig. 6B). Hypertrophied myocytes revealed a decreased ratio of reticulate to punctate mitochondrial structures $(7.05 \pm 1.35$-fold) compared with control myocytes that was significantly restored in $P P A R \alpha$-overexpressed hypertrophied myocytes $(3.55 \pm 0.38$ fold; Fig. 6B). The aspect ratio $(A R)$ and the form factor $(F)$ revealed significant fragmentation of mitochondrial networks during hypertrophy $(A R: 5.50 \pm 0.47$-fold and $F: 4.86 \pm 0.33$ fold) with respect to control that was also restored significantly in $P P A R \alpha$-overexpressed hypertrophied myocytes (AR: 3.07 \pm 0.22 -fold and $F: 2.17 \pm 0.17$-fold; Fig. 6B).

Targeted overexpression of nanotized $P P A R \alpha$ in ligated cardiac tissue revealed substantial reduction of blurred cristae, mitochondrial swelling, and fragmentation (Fig. 6C), along with significant increase of mitochondrial diameter $(1.85 \pm 0.21-$ fold) and average mitochondrial area (2.21 \pm 0.26 -fold; Fig. 6C) compared with empty vector-targeted ligated group, as revealed by transmission electron microscopic analyses.

\section{PPAR $\alpha$ ameliorates myocyte oxygen consumption and adenosine triphosphate generation during hypertrophy}

Ligated rat myocardium showed significant downregulation of baseline cellular oxygen consumption rate (OCR) (1.44 \pm 0.11 -fold) and adenosine triphosphate (ATP) generation (1.63 \pm 0.14 -fold) compared with sham control that was significantly restored by nanotized overexpression of $P P A R \alpha$ in ligated rats (intracellular OCR: 1.22 \pm 0.062 -fold and ATP generation: $1.29 \pm 0.15$-fold) and compared with empty vectortreated ligated group (Fig. 7A, B).

\section{Discussion}

The present study reports amelioration of apoptotic load and improvement of cardiac function by myocardiumtargeted nanotized overexpression of PPAR $\alpha$ during

FIG. 6. Targeted overexpression of PPAR $\alpha$ improves mitochondrial structure during hypertrophy. (A) RT-PCR showing mitochondrial copy number in different experimental groups both in vitro and in vivo, as revealed by expression of mitochondrial $m t C O 1$ gene with respect to single copy nuclear gene Ndufvl. For in vitro: lanes C, control myocytes; A, AngII-treated myocytes; A+P, PPAR $\alpha$-overexpressed hypertrophied myocytes; A+C646, C646-treated hypertrophied myocytes and A+p53si, p53 siRNA-treated hypertrophied myocytes; and for in vivo: lanes $\mathrm{S}$, sham operated control heart; L, ligated rat heart; L+P, myocyte-targeted PPAR $\alpha$-overexpressed ligated heart; and L+p53si, myocyte-targeted $p 53$ siRNAtreated ligated heart. Control and hypertrophy groups were treated with DMSO or NS siRNA or empty plasmid, yielding similar results. $n=5$ for each group. (B) Immunofluorescence study using MitoTracker ${ }^{\circledR}$ Red showing mitochondrial network shape in different experimental groups. Hypertrophied myocytes showing substantially increased punctate to reticulate mitochondrial networks (ii, v) compared with control cells (i, iv). $P P A R \alpha$-overexpressed hypertrophied cells showing restoration of reticulate mitochondrial pattern (iii, vi). Control and hypertrophied cells were treated with empty plasmid. $n=3$ for each group. Scale bar $=20 \mu M, 100 \times$ magnification for all panels. Corresponding graph showing levels of $\%$ mitochondrion shape transition. $*^{*} p<0.01$ with respect to control; ${ }^{\#} p<0.05$ with respect to AngII. Furthermore, $A R$ and $F$ transitions were also calculated and represented in a graphical form. $* * * p<0.001$ with respect to control; ${ }^{\dagger \dagger} p<0.01$ with respect to AngII. (C) Transmission electron microscopy showing betterment of mitochondrial ultrastructure in ligated group (panel ii) compared with $P P A R \alpha$-overexpressed ligated rats (iii). Scale bar $=1 \mu \mathrm{m}, 5000 \times$ magnification (panels i-iii). $n=5$ for each group. Control and ligated groups were treated with empty plasmid. Corresponding graphs showing mitochondrial diameter and average mitochondrial area in vivo from different treatment groups. $n=5$, for each group. $* * p<0.01$ with respect to control; ${ }^{\dagger} p<0.01$ with respect to ligated group. AR, aspect ratio; F, form factor. To see this illustration in color, the reader is referred to the web version of this article at www.liebertpub.com/ars 

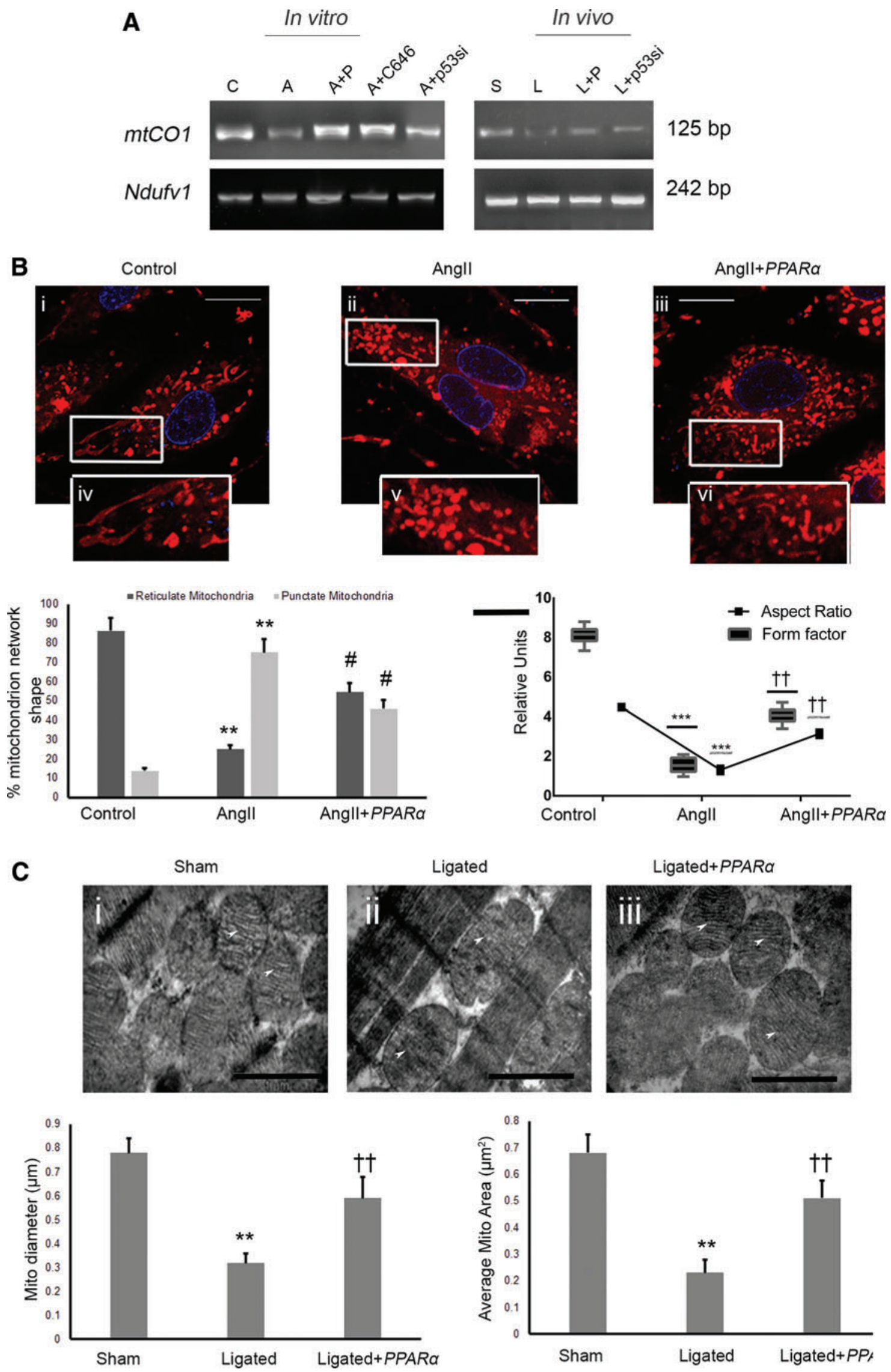

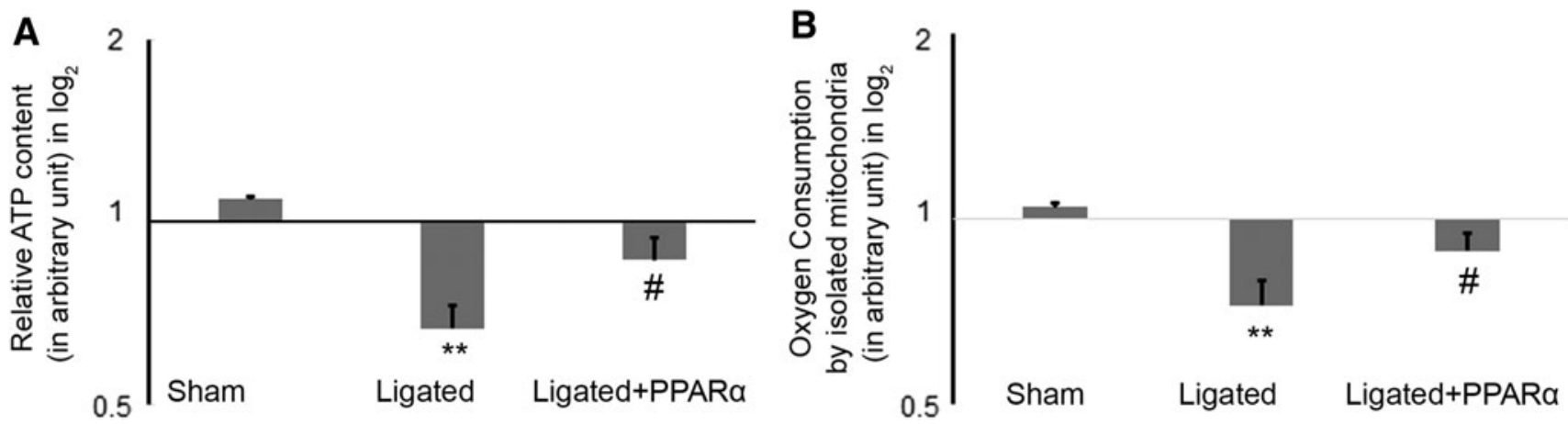

FIG. 7. Targeted overexpression of PPAR $\alpha$ mediates restoration of mitochondrial function in hypertrophied myocardium. (A) Graph showing relative changes in myocardial ATP generation from different treatment groups in vivo. Control and ligated groups were treated with empty plasmid. All values were $\log _{2}$ transformed. $* * p<0.01$ with respect to sham; ${ }^{\#} p<0.05$ with respect to ligated; $n=5$, for each group. (B) Graph showing relative changes in myocardial oxygen consumption rate from different treatment groups in vivo. Control and ligated groups were treated with empty plasmid. All values were $\log _{2}$ transformed. $* * p<0.01$ with respect to sham; ${ }^{*} p<0.05$ with respect to ligated; $n=5$, for each group. ATP, adenosine triphosphate.

pathological hypertrophy. According to previous reports, MHC- $P P A R \alpha$ transgenic animals showed diabetic cardiomyopathy accompanied with altered myocardial metabolic profile and lipid imbalance $(21,54)$, as well as high level of prenatal $\operatorname{PPAR} \alpha$ activation might lead to postnatal lethality (1). Our study aims to probe into the effect of the targeted transient overexpression strategy of PPAR $\alpha$ in hypertrophied myocardium. In this study, we showed reduced bystander effect in other organs by the targeted nanopolymer. Myocytetargeted overexpression of PPAR $\alpha$ was achieved by a CMCP (Fig. 1B-G; Supplementary Figs. S1, S5B, C). M-mode echocardiographic analyses in such $P P A R \alpha$-overexpressed ligated rats showed significantly improved cardiac function (Fig. 2D; Supplementary Fig. S3; Supplementary Table S1) via effective inhibition of p53 acetylation (Fig. 3A), improved mitochondrial structure and function (Figs. 5-7), and attenuation of myocyte apoptosis (Fig. 2E, F; Supplementary Fig. S6B). This report shows for the first time that targeted overexpression of $P P A R \alpha$ in hypertrophied myocytes results in efficient amelioration of oxidative stress, thereby regressing hypertrophic stimuli and improving functional capacity of the diseased myocardium.

Studies have suggested that cardiac hypertrophy reduces ATP generation and oxygen consumption $(59,70)$, as has been reported in PPAR $\alpha$-deficient animals with blunted FAO gene expression $(4,9,36)$. Previous works have also reported that maintenance of FAO during cardiac pathophysiology improved cardiac function $(10,32)$. Besides, active forms of PPAR $\alpha$ and ATGL are reported to be mutual agonists in restoration of mitochondrial defects $(12,60)$ and PPAR $\alpha$ agonists have been shown to attenuate stress-activated kinase-mediated apoptotic signaling pathways (19). Despite these findings, molecular mechanism of PPAR $\alpha$-mediated restoration of mitochondrial function in compromised heart still remains obscure.

In our study, significant downregulation of PPAR $\alpha$ during cardiac hypertrophy supported the previous findings (Fig. 1A; Supplementary Fig. S5A). The pathophysiological outcome of $P P A R \propto$ transgenesis during cardiac hypertrophy emerged as a proposition toward empirical analyses. The gene therapy involved stable encapsulation of the overexpression vector by the myocardium-targeted $\mathrm{CMCP}$ nanopolymer. The targeted nanoconstruct (CMCP) showed stable encapsulation by gel retardation analysis, in which an encapsulation weight ratio of 1:50 (CMCP:pcDNA) showed significant plasmid retention and protection from serummediated nuclease degradation (Fig. 1B; Supplementary Fig. S1B). The plasmid-encapsulated CMCP revealed homogenous size distribution by dynamic light scattering (DLS) analysis and scanning electron microscopy, while positive charge was revealed by $\zeta$ potential analysis (Fig. 1C; Supplementary Fig. S1A).

Overall, the nanoconstruct signified an increased in vivo retention, while the cellular uptake of biomaterials possibly occurred via an endolysosomal pathway (57). Overexpression of PPAR $\alpha$ by CMCP encapsulation showed stable expression of associated his-tag in vitro. CMCP-mediated $P P A R \propto$ gene delivery showed higher expression of his-tag in myocardium compared with bystander organs in vivo by Western blot analysis, thereby confirming the relative uptake and targeted bioavailability of the PPAR $\alpha$ nanoconstruct delivery system (Fig. 1D, E; Supplementary Fig. S5C). Moreover, selective targeting by the 20 -mer myocyte targeting peptide conjugated to the chitosan nanoconstruct was confirmed by colocalization of his-tag in myocytes counterstained with $\alpha$-sarcomeric actin in vivo (Fig. $1 \mathrm{~F}, \mathrm{G}$ ).

The CMCP nanoconstruct-mediated myocyte-targeted transgenesis and overexpression of PPAR $\alpha$ led to concomitant upregulation of downstream signaling molecules, namely, PGC1 $\alpha$, ATGL, and CPT1 $\alpha$ (Supplementary Fig. S2), involved in utilization of fatty acid and subsequent mitochondrial uptake of fatty acid for FAO $(4,27,70)$. Interestingly, nanoconstructmediated targeted overexpression of PPAR $\alpha$ during cardiac hypertrophy led to significant attenuation of hypertrophic marker genes, reduced cardiomyocyte cross-sectional area, and improved cardiac function compared with the respective hypertrophy group (Fig. 2A-D; Supplementary Fig. S3; Supplementary Table $\mathrm{S} 1$ ). Furthermore, overexpression of $P P A R \alpha$ in hypertrophy samples showed significant downregulation of caspase- 3 activity and reduced mitochondrial cytochrome-C 


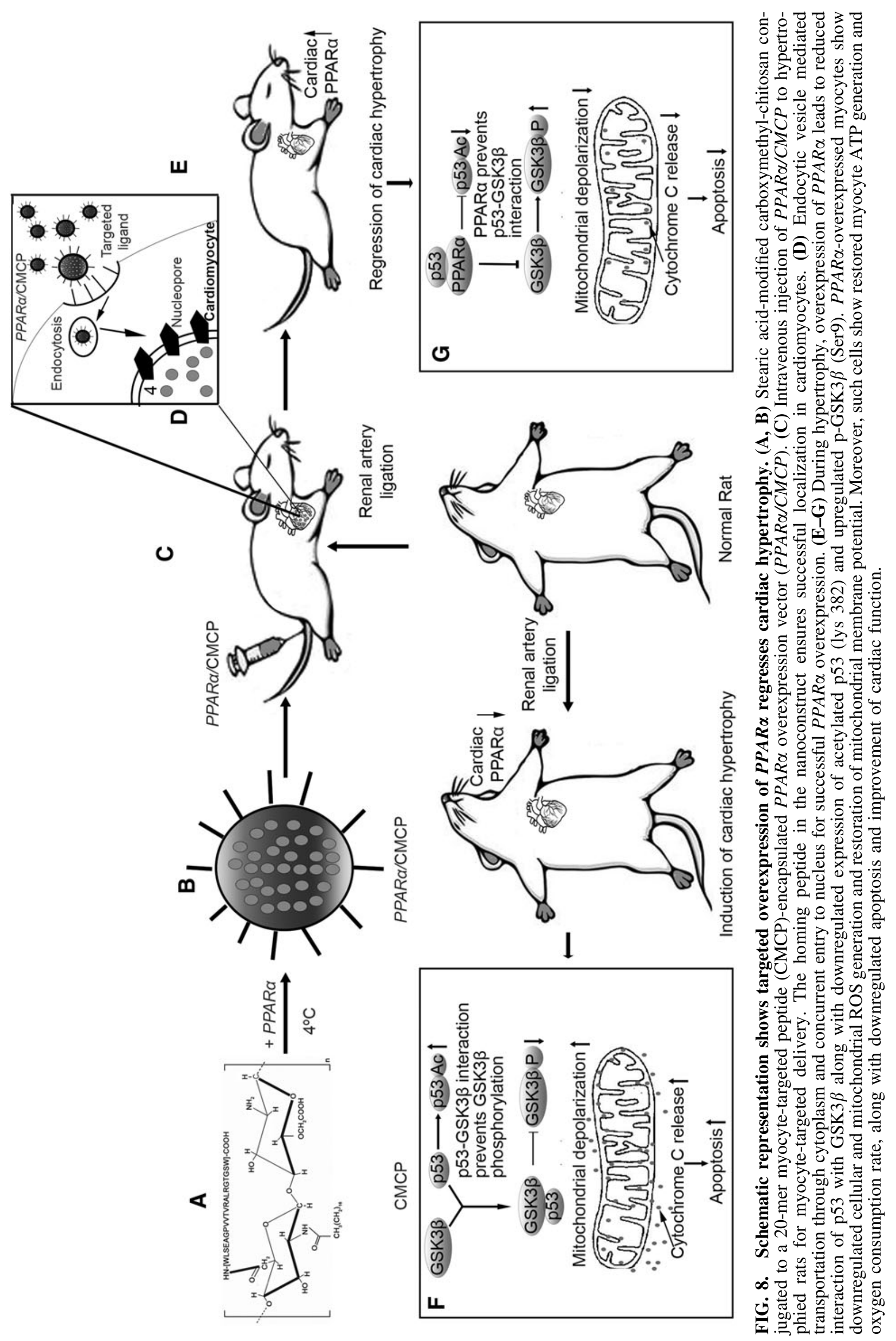


release, suggesting reduced mitochondria-mediated apoptosis in the $P P A R \alpha$-overexpressed hypertrophied myocardium (Fig. 2E, F; Supplementary Fig. S6B).

Several reports have associated mitochondrial apoptosis with p53 acetylation during cardiac hypertrophy (40). Although our study revealed no significant change in p53 expression between myocyte-targeted PPAR $\alpha$-overexpressed hypertrophy groups compared with the empty plasmid-treated hypertrophy group, p53 acetylation (lys 382) was significantly downregulated in such samples compared with the hypertrophy group (Fig. 3A). Reportedly, acetylation of p53 protects it from mouse double minute 2 homolog (MDM2)-mediated ubiquitination (37). In hypertrophied myocytes, higher expression of MDM2 facilitates downregulation of PPAR $\alpha$ (data not shown); moreover, MDM2-mediated proteasomal degradation of PPAR $\alpha$ has also been associated in other cell types $(7,24)$. In line, p53 activates MDM2 transcription (3) and as a result knockdown of p53 during hypertrophy results in subsequent transcriptional downregulation of MDM2. Thereby, C646-mediated blockade to p53 acetylation and p53 knockdown showed an upregulated expression of PPAR $\alpha$. Next, the study focused on exploring the possible molecular mechanisms involved in downregulation of p53 acetylation during PPAR $\alpha$ overexpression in hypertrophy groups.

To understand this conundrum, coimmunoprecipitation and reverse coimmunoprecipitation analyses with "his" and "flag" tag antibodies were performed in myocytes transfected with $p 53-\mathrm{CMV}$ and $P P A R \alpha-\mathrm{pcDNA}$, showing substantial interaction of $\operatorname{PPAR} \alpha$ with p53 (Fig. 3B). Furthermore, the interaction of PPAR $\alpha$ to the C-terminal domain of p53 was revealed by the interaction of PPAR $\alpha$ (his-tag) with different domains of p53 (flag-tag). Notably, flag-tag coimmunoprecipitation analyses followed by histag immunoblotting showed no interaction of 1-100 aa and 1-300 aa domains of p53 with $\operatorname{PPAR} \alpha$, while full-length p53 (1-391 aa) showed significant interaction with $\operatorname{PPAR} \alpha$ compared with empty plasmid-treated myocytes (Fig. 3C), thereby indicating interaction of PPAR $\alpha$ with $301-391$ aa region of $\mathrm{p} 53$.

The specific region is also important for the interaction of p53 with its transcriptional machinery components, including TATA-binding protein and its associated factors ([TAFs] 49), and with its transcriptional coactivator p300 (64). This report brings us to the "masking model" hypothesis, through which the binding of PPAR $\alpha$ to p53 conceals its C-terminal domain (53). The association of wild-type p53 in apoptosis is explicitly known $(16,25)$, moreover, studies have also implied the involvement of the p53 C-terminal domain in regulation of apoptosis $(29,65)$. Furthermore, interaction of GSK $3 \beta$ with p53 at 364-373 aa of the basic domain determines activity of both $(67,68)$ and is crucial in determining cell fate by modulating mitochondrial apoptosis (42). Moreover, myocyte-targeted overexpression of $P P A R \alpha$ during cardiac hypertrophy showed reduced binding of GSK $3 \beta$ with p53 by coimmunoprecipitation analyses, as was observed by the C646 treatment. These data thus suggest the importance of p53 acetylation (lys 382) in GSK3 $\beta$-p53 binding. Also, FRAP analyses confirmed that increased PPAR $\alpha$-p53 binding in hypertrophied myocytes abrogated the interaction of $\mathrm{p} 53$ with GSK3 $\beta$ (Fig. 4A, B; Supplementary Fig. S8A). Thus, interaction of $\operatorname{PPAR} \alpha$ with p53 at the C-terminal domain might inhibit the amino acid residues of that particular region for various post-translational modifications and interaction with other proteins $(34,56)$.

Markedly, the lipid binding property of p53 by the Cterminal domain is critical in mitochondrial p53 localization and resultant cell death pathways $(38,50)$. The active form of GSK $3 \beta$ within mitochondria induces oxidative stress injury and opening of mitochondrial permeability transition pore that leads to an upregulated cellular ROS generation (2). In our study, $P P A R \alpha$-overexpressed and C646-treated hypertrophied myocytes showed upregulated expression of GSK3 $\beta$ (Ser9) within mitochondrial compartments (Fig. 4C; Supplementary Fig. S8B). These data suggested that reduced acetylation of $\mathrm{p} 53$ promotes GSK $3 \beta$ inactivation, which renders improvement of mitochondria and subsequent cardioprotection during hypertrophic pathophysiology (30, $42,63)$. The concomitantly increased expression of GSK3 $\beta$ (Ser9) phosphorylation and decreased expression of p53 acetylation were found to be associated with a downregulated ROS load in PPAR $\alpha$-overexpressed hypertrophied myocytes compared with untreated hypertrophied cells (Fig. 4D). Cellular activation of various protein kinases by redox-dependent mechanisms may be instrumental toward inactivation of GSK $3 \beta$ by phosphorylation at the Ser9 residue $(12,14,20,23,30)$. Thus, the role of PPAR $\alpha$ overexpression in the hypertrophied cells is instrumental toward lowering the apoptotic burden; however, the functional betterment of the myocytes required further exploration of the mitochondrial structure and function.

The failing heart has been associated with induction of $\mathrm{O}_{2}{ }^{-}$ flashes that transiently decrease myocyte $\Delta \Psi \mathrm{m}$, which further leads to qualitative and quantitative mitochondrial derangements such as energetic dysfunction, swelling, and rupture (26, $28,35,66)$, altogether reducing the capacity of heart to convert chemical energy into mechanical work (51). Our study revealed that $P P A R \alpha$-overexpressed hypertrophied myocytes reduced mitochondrial $\mathrm{O}_{2}{ }^{-}$radical accumulation and restored $\Delta \Psi \mathrm{m}$ compared with hypertrophied cells (Fig. 5A, B; Supplementary Fig. S4A). Myocyte-targeted overexpression of $P P A R \alpha$ also reduced mitochondrial Drp1 expression that regulates cytochrome-C release during apoptosis (31), along with downregulated expression of Bax and Bid in hypertrophied myocardium (Fig. 5C). In addition, targeted overexpression of $P P A R \alpha$ restored mitochondrial copy number and Tfam expression (Figs. 5D and 6A), reportedly downregulated during cardiac hypertrophy (15). Myocyte-targeted $P P A R \alpha$-overexpressed hypertrophy groups showed substantial restoration of mitochondrial structural integrity marked by increased ratio of reticulate to punctate mitochondrial networks, increased mitochondrial diameter and mitochondrial area, and partial restoration of mitochondrial $A R$ and $F$ compared with hypertrophy groups (Fig. 6B, C). This led to mitochondrial functional restoration as revealed by improved ATP generation and OCR (Fig. 7A, B) in myocyte-targeted $P P A R \alpha$-overexpressed ligated groups compared with ligated groups alone. Thus, restored energy generation stabilized mitochondrial oxidative metabolism and oxidative phosphorylation in the $P P A R \alpha$-overexpressed pathological cardiac hypertrophy groups.

Taken together, CMCP nanoconstruct-mediated myocytetargeted overexpression of PPAR $\alpha$ upregulated PPAR $\alpha$ expression level during pathological hypertrophy that resulted in regressed apoptotic stress and altered the balance of 
apoptosis toward mitochondrial biogenesis by increased mitochondrial structural restitution against oxidative stress. Moreover, targeted delivery of PPAR $\alpha$ ensures minimal bystander activity of this protein, thereby underlining the therapeutic benefit of myocyte-targeted overexpression of PPAR $\alpha$ during cardiac pathophysiology. Altogether, this study for the first time delineates PPAR $\alpha$ as a novel "tinkerer" in modulation of $\mathrm{p} 53$ and GSK $3 \beta$ axis toward prevention of mitochondrial apoptosis and restoration of cardiac function in ligated rats (Fig. 8).

\section{Materials and Methods}

\section{Animals used}

The 24-week-old male Wistar rats (Rattus norvegicus; $n=50)$ used in this study were procured from NIN, Hyderabad, India, and conform with the Guide for the Care and Use of Laboratory Animals published by the U.S. National Institute of Health (NIH Publication No. 85-23, revised 1996) and approved by the Institutional Animal Ethics Committee, University of Calcutta (Registration No. 885/ac/05/CPCSEA), registered under "Committee for the Purpose of Control and Supervision of Experiments on Laboratory Animals,", Ministry of Environment and Forest, Government of India.

\section{Animal model and treatment}

In vivo cardiac hypertrophy was generated by ligating the right renal artery of 24-week-old anesthetized rats (43). Animals were kept under optimum conditions for 14 days and sacrificed on the 15th day after surgery. Sham operated control rat groups underwent a similar surgical procedure without ligation. Heart, kidney, brain, liver, and lung tissues were collected for further experimentation. The HW/BW ratio, in $\mathrm{mg} / \mathrm{g}$, was calculated from heart tissue samples of different rat groups. Rats $(n=5)$ were used for each experimental group.

\section{Isolation, culture, and treatment of myocytes}

Hearts from 2- to 3-day-old neonatal rat pups were dissected out and minced in $10 \%$ fetal bovine serum medium. Collagenase media $(80 \mathrm{U} / \mathrm{mL})$ were added to minced hearts and stirred for $5 \mathrm{~min}$ at room temperature, this was repeated four times. The supernatants were centrifuged to pellet down the cells, which were resuspended in fresh media and incubated for $45 \mathrm{~min}$. Finally, cells were plated in laminin (Sigma-Aldrich, St. Louis, MO)-coated plates and coverslips. Approximately $90 \%$ pure isolated cardiomyocytes were confirmed by staining with sarcomeric $\alpha$-actin antibody. Myocytes were serum starved with Dulbecco's modified Eagle's medium (DMEM) for $12 \mathrm{~h}$, before experimentation. Isolated serum-starved myocytes were treated for $24 \mathrm{~h}$ with $10^{-8} \mathrm{~mol} / \mathrm{L}$ (Sar ${ }^{1}$ )-AngII (Bachem, Torrance, CA) (52). Myocytes were treated with p300-HAT activity inhibitor C646 (10 $\mu \mathrm{M}$; SigmaAldrich) during AngII treatment for blocking p300-mediated acetylation of p53 (58).

\section{Plasmid construction}

Full-length coding DNA sequence of PPAR $\alpha$ (Reference Sequence: NM_013196.1) was cloned in pcDNA6/V5-His B mammalian expression vector (Thermo Fisher, Waltham,
MA) with C-terminal his-tag as described previously (17). Full-length p53 (NM_030989.3) and its domains (1-100, 1300, and 1-391 aa) were cloned in pFLAG-CMV ${ }^{\mathrm{TM}}-3$ expression vector (Sigma-Aldrich) with N-terminal flag-tag as described previously (6). All the clones were confirmed by sequencing (3730 DNA Analyzer; Applied Biosystems, Foster City, CA).

\section{Preparation and biophysical characterization of targeted PPAR $\alpha$ gene delivery system}

The stearic acid-modified carboxymethyl-chitosan conjugated to the 20-mer peptide was synthesized according to Rana et al. (57), with minor modifications. Briefly, lowmolecular-weight chitosan (Sigma-Aldrich) was used to produce CMC. The unreacted amine groups after modification with stearic acid were protected by di-tert-butyl dicarbonate $\left(\mathrm{BOC}_{2} \mathrm{O}\right.$; Sigma-Aldrich). A mixture of $1 \mathrm{mM}$ 1-ethyl-3-(3-dimethylaminopropyl) carbodiimide (EDC) and $1 \mathrm{~m} M \mathrm{~N}$-hydroxysuccinimide (Thermo Fisher) was slowly added onto $\mathrm{BOC}_{2} \mathrm{O}$-protected stearic acid-modified CMC along with dropwise addition of the peptide $[\mathrm{H}]-$ WLSEAGPVVTVRALRGTGSW-[OH] (Sigma-Aldrich) under continued stirring for $72 \mathrm{~h}$ at $4^{\circ} \mathrm{C}$ to ensure proper binding of peptide with CMC. The solution was centrifuged to precipitate peptide-conjugated CMC and then lyophilized. Finally, trifluoroacetic acid was added for $\mathrm{BOC}_{2} \mathrm{O}$ deprotection. PPAR $\alpha$ cloned in pcDNA6/V5-His B mammalian expression vector was added to the $\mathrm{CMCP}$ at different weight ratios and incubated for $2 \mathrm{~h}$ at $4^{\circ} \mathrm{C}$ under constant shaking conditions ( $400 \mathrm{rpm})$, followed by centrifugation at $13,000 \mathrm{rpm}$ for $20 \mathrm{~min}$ at $4^{\circ} \mathrm{C}$, filtered, and lyophilized. $P P A R \alpha$-encapsulated CMCP at different weight ratios was electrophoresed.

$P P A R \alpha$-encapsulated CMCP dissolved in Na-acetate buffer was mildly sonicated and then centrifuged (4000 rpm at $4{ }^{\circ} \mathrm{C}$ ) for $5 \mathrm{~min}$ and characterized by DLS (DynaPro NanoStar ${ }^{\mathrm{TM}}$; Wyatt Technology, Santa Barbara, CA), $\zeta$

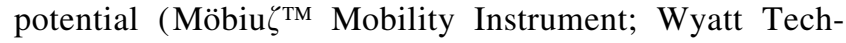
nology), and scanning electron microscopy (Hitachi VPSEM S-3400N).

The amount of DNA encapsulated within CMCP (DNA loading efficiency) was assessed by quantifying the concentration of plasmid DNA from a standard curve and encapsulation efficiency was calculated by dividing plasmid DNA entrapped within CMC peptide by plasmid DNA added for conjugation.

CMCP-entrapped pcDNA complex was incubated with $20 \%(\mathrm{v} / \mathrm{v})$ rat serum diluted with DMEM (13). The complex was treated after regular time intervals in different tubes, with heparin sodium at a concentration of $30 \mathrm{IU} / \mu \mathrm{g}$ of pcDNA.

\section{Treatment with targeted gene delivery system}

Myocytes were transfected with 500 pmoles of p53 siRNA (FlexiTube siRNA; Qiagen, Germantown, MD) or negative control siRNA (AllStars Negative Control siRNA, Catalog No. 1027280; Qiagen) by a nanopolymeric encapsulation of CMCP (57). CMCP-encapsulated plasmids (2-4 $\mu \mathrm{g})$ were transfected to myocytes. For in vivo experiments, plasmid and siRNA with CMCP encapsulation were intravenously injected via tail vein at a dose of $2 \mathrm{mg} / \mathrm{kg}$ of BW/day to renal 
artery ligated rats, on alternate days, starting from 8th day till 14 th day of ligation.

\section{Protein isolation}

After the experimental period, hearts were dissected out and perfused in chilled phosphate-buffered saline (PBS) for all in vivo experiments. For all in vitro studies, isolated myocytes after treatment were washed in chilled PBS. Protein from cells or tissues was isolated using protein extraction buffer [7 M urea, 4\% (w/v) 3-[(3-cholamidopropyl) dimethylammonio]-1-propanesulfonate hydrate, $2 \mathrm{M}$ thiourea, $1 \times$ ethylenediaminetetraacetic acid-free protease inhibitor cocktail, and phosphatase inhibitor cocktail (Roche, San Francisco, CA)] as described previously (44). The protein supernatants were collected from each sample and concentrations were estimated by Bradford assay. Mitochondrial protein fractions from both cell and tissue samples were isolated by the differential centrifugation method as described previously $(11,44)$.

\section{Western blotting and coimmunoprecipitation analyses}

Proteins from ventricular tissues were extracted and separated by sodium dodecyl sulfate (SDS)/polyacrylamide gel electrophoresis before being transferred to polyvinylidene difluoride+ membrane. After blocking with 5\% nonfat dry milk, membranes were incubated with primary antibodies in $5 \%$ bovine serum albumin solution at $4^{\circ} \mathrm{C}$ overnight. After washing, membranes were incubated with appropriate horseradish peroxidase-conjugated secondary antibodies (Thermo Fisher, Waltham, MA) at room temperature for $1 \mathrm{~h}$. Finally, the immunoreactive bands were visualized by the enhanced chemiluminescence kit (Millipore, Billerica, MA) (11).

Coimmunoprecipitation was done following the manufacturer's protocol Pierce Coimmunoprecipitation Kit (Thermo Fisher). Briefly, proteins were incubated with fast flow protein $G$ or protein A Sepharose beads and centrifuged to eliminate nonspecifically bound proteins (17). Concentration of the precleared proteins was estimated and $200 \mu \mathrm{g}$ of protein was immunoprecipitated using primary antibodies of anti-p53, anti-his, and anti-flag. The immunoprotein complex was again incubated with protein $G$ or protein A Sepharose beads. Attached proteins were then eluted from beads in $1 \%$ SDS buffer followed by immunoblotting with specific monoclonal antibodies (17). Normalization was done by immunoblotting using the same antibodies (Supplementary Table S2).

\section{Reverse transcription-polymerase chain reaction}

Total RNA and DNA from cells/tissues were isolated using TRIzol reagent (Thermo Fisher) following the manufacturer's protocol. Two micrograms of total RNA was used to make complementary DNA using cloned avian myeloblastosis virus First Strand cDNA Synthesis Kit (Thermo Fisher) and polymerase chain reaction (PCR) was done for the target genes using specific primers against $A N F$ and $\beta$ $M H C$ (11). Five hundred nanograms of total DNA was used for mtDNA copy number determination by PCR. Relative change in mtDNA copy number was determined by $m t C O 1$ mitochondrial gene (forward: 5'-TGCTAGCCGCAGGCAT TAC-3'; reverse: 5'-GGGTGCCCAAAGAATCAGAAC-3') and single copy nuclear gene $N d u f v l$ (forward: $5^{\prime}$-CTTC
CCCACTGGCCTCAAG-3'; reverse: 5'-CCAAAACCCAG TGATCCAGC-3') (60).

\section{Immunofluorescence analyses}

Frozen ventricular tissue sections $(4 \mu \mathrm{m})$ were prepared using CM 1850 cryostat (Leica, Germany). Tissue sections and cells plated on laminin-coated coverslips were stained with primary antibodies to anti- $\alpha$-sarcomeric actin, antiTfam, anti-caspase-3 cleaved fragment, anti-GSK3 $\beta$, anti p53, and anti-his followed by incubation with specific labeled secondary antibodies (Molecular Probes, Eugene, OR; Supplementary Table S2) (62). After mounting with Vectashield [with 4',6-diamidino-2-phenylindole (DAPI)] (Vector Laboratories, Burlingame, CA), cells and tissues were visualized under FV1200 confocal microscope (Olympus, Japan).

\section{FRAP analyses}

Transfected and untransfected hypertrophied myocytes were stained with GSK3 $\beta$-TRITC and p53-FITC. As TRITC and FITC work as a donor/acceptor in FRAP assay, TRITC was subjected to $50 \%$ bleaching, and FITC intensity in a single cell was measured. FRET efficiency was calculated from the difference between the postbleaching and prebleaching donor intensities (delta donor) using FV1200 confocal microscope (18).

\section{Analysis of $\Delta \Psi m$}

Loss of $\Delta \Psi \mathrm{m}$ was assessed on myocytes by incubating myocytes with tetraethyl benzimidazolyl carbocyanine iodide (JC-1; Thermo Fisher), a cationic dye that accumulates in energized mitochondria after treatment. Cells were incubated with $5 \mu \mathrm{M} \mathrm{JC}-1$ for $15 \mathrm{~min}$ at $37^{\circ} \mathrm{C}$ to load the cells with dye. Myocytes after treatment were washed with $1 \times \mathrm{PBS}$ mounted on slides with Vectashield before scanning under confocal microscope (41). Stained myocytes were individually imaged using the $100 \times$ objective; the monomeric form of JC-1 was excited using the 488-nm laser, and the aggregate form was excited using the 543-nm laser. Acquired images were analyzed using ImageJ, and the fluorescence intensity for each JC-1 form was determined for multiple regions of interest (ROIs).

\section{MitoTracker labeling}

Myocytes were treated with 500 nM MitoTracker Red FM (Thermo Fisher) for $15 \mathrm{~min}$ at $37^{\circ} \mathrm{C}$, after which they were incubated in dye-free medium (39). The MitoTracker Red fluorophore molecules accumulated in mitochondria, which was determined by exciting with the 561-nm laser and the fluorescence intensity was determined from multiple ROIs per myocyte and analyzed with Image J software.

Mitochondrial morphological characteristics were quantified for $A R$ (ratio between major and minor axes of an ellipse equivalent to the mitochondrion, measure of mitochondrial length) and $F$ (perimeter ${ }^{2} / 4 \pi \cdot$ area, a combined measure of both mitochondrial length and degree of branching). $A R$ is a measure of mitochondrial length and reflects the "length-towidth ratio"; $F$ is a combined measure of both mitochondrial length and degree of branching and reflects the complexity and branching aspect of mitochondria. Both $A R$ and $F$ are independent of image magnification and have a minimal 
value of 1 (corresponding to a circular mitochondrion). Therefore, one was routinely subtracted from the experimental values obtained for $A R$ and $F(33,55)$.

\section{Assessment of ROS levels}

Myocytes after respective treatments were incubated with $50 \mu M 2^{\prime}, 7^{\prime}$-dichlorofluorescin diacetate (DCFDA) (Abcam, Cambridge, MA) at $37^{\circ} \mathrm{C}$ in the dark for $45 \mathrm{~min}$. The treatment media were completely removed before DCFDA staining. Cells were then washed with $1 \times$ buffer and fluorescence intensities were acquired by fluorimetry at an excitation wavelength of $485 \mathrm{~nm}$ and emission wavelength of $535 \mathrm{~nm}$. Tert-butyl hydrogen peroxide was used as positive control in ROS assay (57).

\section{Mitochondrial ROS levels}

MitoSOX Red mitochondrial $\mathrm{O}_{2}^{-}$indicator (Thermo Fisher) was used to monitor changes in mitochondrial $\mathrm{O}_{2}{ }^{-}$ production by confocal microscopy (69). Myocytes were loaded with MitoSOX Red $(5 \mu \mathrm{m})$ for $30 \mathrm{~min}$ at room temperature, followed by $1 \times$ PBS wash. MitoSOX Red was excited at $488 \mathrm{~nm}$ and measured at an emission of $585 \mathrm{~nm}$.

\section{Caspase- 3 activity assay}

Caspase-3 activity from ventricular tissue of all experimental groups was determined using the ApoAlert Caspase-3 Assay Kit (Clontech Q25 Laboratories, Mountain View, CA) following the manufacturer's protocol (11).

\section{ATP assay and OCR in vivo}

Ventricular tissue samples were lysed in ATP releasing buffer as described previously (39) and ATP levels were measured using the ATP Determination Kit (Molecular Probes ${ }^{\circledR}$; Life Technologies, Camarillo, CA) as per the manufacturer's protocol. ATP content was calculated as nanomolars of ATP per microgram of protein.

Oxygen consumption by mitochondria isolated from cardiac muscle was measured in a Hansatech oxygraph (Hansatech, Norfolk, UK) as described previously (47). OCRs were calculated as $\mathrm{nmol} / \mathrm{mg}$ tissue $/ \mathrm{min}$.

\section{Histology (hematoxylin and eosin)}

Heart tissue sections from animals of each group were fixed with acetone and methanol (1:1) and stained with hematoxylin and eosin for measurement of cardiomyocyte cross-sectional area (CSA) under microscope (Nikon Microscope, Singapore). Images were digitized and measured by Nikon NIS BR (57).

\section{Electron microscopy}

Cardiac tissue samples were fixed and embedded as described previously (45). Thin sections (80-90 nm) were briefly washed and stained with $2.5 \%$ uranyl acetate, and visualized under Morgagni 268D transmission electron microscope (Fei Company Q24, The Netherlands).

\section{M-mode echocardiography}

Cardiac function of lightly sedated animals from all groups was measured by M-mode analyses on a transthoracic study at the 15 th day before euthanization. Digitized images were obtained using an ultrasound system (Vivid S5 System; GE Healthcare, Chicago, IL) for calculation of the following parameters, namely, LVDd, LVDs \%FS, LvAWDd, LvAWDs, LvPWDd, and LvPWDs (44, 57).

\section{Statistical analyses}

All results are expressed as mean \pm standard error of three independent experiments. Data were analyzed by Student's $t$ test (comparison of two groups) and one-way analysis of variance followed by Tukey's test (comparison of multiple groups) using GraphPad Prism (Version 6.01; GraphPad Software, La Jolla, CA). Results with $p$-value $<0.05$ were considered significant $(18,58)$.

\section{Acknowledgments}

The authors sincerely acknowledge Barbara Smith (Coventry University, Coventry, UK) for carefully editing the article. This work was funded by the Department of Biotechnology (DBT) (Grant Nos. BT-PR3709/BRB/10/980/ 2011 and BT/PR7016/NNT/28/641/2012) and Department of Science and Technology (DST) (Grant No. SB/SO/HS-148/ 2013), Government of India, to Dr. S. Sarkar and DST-FIST Program (Grant No. SR/FST/LS1-438/2000(G)).

\section{Ethical Approval}

This article does not contain any studies with human participants performed by any of the authors. All procedures performed in studies involving animals were in accordance with the ethical standards of the institution or practice at which the studies were conducted.

\section{Author Disclosure Statement}

No competing financial interests exist.

\section{References}

1. Abbott BD, Wolf CJ, Schmid JE, Das KP, Zehr RD, Helfant L, Nakayama S, Lindstrom AB, Strynar MJ, and Lau C. Perfluorooctanoic acid-induced developmental toxicity in the mouse is dependent on expression of peroxisome proliferatoractivated receptor-alpha. Toxicol Sci 98: 571-581, 2007.

2. Balaban RS, Nemoto S, and Finkel T. Mitochondria, oxidants, and aging. Cell 120: 483-495, 2005.

3. Barak Y, Gottlieb E, Juven-Gershon T, and Oren M. Regulation of $\mathrm{mdm} 2$ expression by $\mathrm{p} 53$ : alternative promoters produce transcripts with nonidentical translation potential. Genes Dev 8: 1739-1749, 1994.

4. Barger PM, Brandt JM, Leone TC, Weinheimer CJ, and Kelly DP. Deactivation of peroxisome proliferator-activated receptor- $\alpha$ during cardiac hypertrophic growth. $J$ Clin Invest 105: 1723-1730, 2000.

5. Beurel E and Jope RS. The paradoxical pro-and antiapoptotic actions of GSK3 in the intrinsic and extrinsic apoptosis signaling pathways. Prog Neurobiol 79: 173189, 2006.

6. Bhowmick R, Halder UC, Chattopadhyay S, Nayak MK, and Chawla-Sarkar M. Rotavirus-encoded nonstructural protein 1 modulates cellular apoptotic machinery by targeting tumor suppressor protein p53. J Virol 87: 68406850, 2013. 
7. Blanquart C, Barbier O, Fruchart JC, Staels B, and Glineur C. Peroxisome proliferator-activated receptor $\alpha(\operatorname{PPAR} \alpha)$ turnover by the ubiquitin-proteasome system controls the ligand-induced expression level of its target genes. $J$ Biol Chem 277: 37254-37259, 2002.

8. Bui AL, Horwich TB, and Fonarow GC. Epidemiology and risk profile of heart failure. Nat Rev Cardiol 8: 30-41, 2011.

9. Campbell FM, Kozak R, Wagner A, Altarejos JY, Dyck JR, Belke DD, Severson DL, Kelly DP, and Lopaschuk GD. A role for peroxisome proliferator-activated receptor $\alpha$ $(\operatorname{PPAR} \alpha)$ in the control of cardiac malonyl-CoA levels reduced fatty acid oxidation rates and increased glucose oxidation rates in the hearts of mice lacking PPAR $\alpha$ are associated with higher concentrations of malonyl-coa and reduced expression of malonyl-CoA decarboxylase. $J$ Biol Chem 277: 4098-4103, 2002.

10. Chambers KT, Leone TC, Sambandam N, Kovacs A, Wagg CS, Lopaschuk GD, Finck BN, and Kelly DP. Chronic inhibition of pyruvate dehydrogenase in heart triggers an adaptive metabolic response. J Biol Chem 286: 11155 11162, 2011.

11. Chatterjee A, Mir SA, Dutta D, Mitra A, Pathak K, and Sarkar S. Analyses of p53 and $\mathrm{NF} \kappa \mathrm{B}$ signaling in modulating the cardiomyocyte fate during hypertrophy. J Cell Physiol 226: 2543-2554, 2011.

12. Chen WL, Chen YL, Chiang YM, Wang SG, and Lee HM. Fenofibrate lowers lipid accumulation in myotubes by modulating the PPAR $\alpha / A M P K / F o x O 1 / A T G L$ pathway. Biochem Pharmacol 84: 522-531, 2012.

13. Chiou HC, Tangco MV, Kormis K, Levine SM, Robertson $\mathrm{D}, \mathrm{Wu} \mathrm{CH}$, and $\mathrm{Wu} \mathrm{GY}$. Enhanced resistance to nuclease degradation of nucleic acids complexed to asialoglycoproteinpolylysine carriers. Nucleic Acids Res 22: 5439-5546, 1994.

14. Cross DA, Alessi DR, Cohen P, Andjelkovich M, and Hemmings BA. Inhibition of glycogen synthase kinase-3 by insulin mediated by protein kinase B. Nature 378: 785789, 1995.

15. Dai DF, Johnson SC, Villarin JJ, Chin MT, Nieves-Cintrón M, Chen T, Marcinek DJ, Dorn GW, Kang YJ, Prolla TA, Santana LF, and Rabinovitch PS. Mitochondrial oxidative stress mediates angiotensin II-induced cardiac hypertrophy and $\mathrm{G} \alpha \mathrm{q}$ overexpression-induced heart failure. Circ Res 108: 837-846, 2011.

16. Das B, Young D, Vasanji A, Gupta S, Sarkar S, and Sen S. Influence of p53 in the transition of myotrophin-induced cardiac hypertrophy to heart failure. Cardiovasc Res 87: 524-534, 2010.

17. Datta R, Bansal T, Rana S, Datta K, Chattopadhyay S, Chawla-Sarkar M, and Sarkar S. Hsp90/Cdc37 assembly modulates $\mathrm{TGF} \beta$ receptor-II to act as a profibrotic regulator of TGF $\beta$ signaling during cardiac hypertrophy. Cell Signal 27: 2410-2424, 2015.

18. Datta R, Bansal T, Rana S, Datta K, Chaudhuri RD, Chawla-Sarkar M, and Sarkar S. Myocyte-derived Hsp90 modulates collagen upregulation via biphasic activation of STAT-3 in fibroblasts during cardiac hypertrophy. Mol Cell Biol 37: e00611-e00616, 2017.

19. De Silva DS, Wilson RM, Hutchinson C, Ip PC, Garcia AG, Lancel S, Ito M, Pimentel DR, and Sam F. Fenofibrate inhibits aldosterone-induced apoptosis in adult rat ventricular myocytes via stress-activated kinase-dependent mecha- nisms. Am J PhysiolHeart CircPhysiol 296: H1983-H1993, 2009.

20. Fang X, Yu SX, Lu Y, Bast RC, Woodgett JR, and Mills GB. Phosphorylation and inactivation of glycogen synthase kinase 3 by protein kinase A. Proc Natl Acad Sci USA 97: 11960-11965, 2000.

21. Finck BN, Lehman JJ, Leone TC, Welch MJ, Bennett MJ, Kovacs A, Han X, Gross RW, Kozak R, Lopaschuk GD, and Kelly DP. The cardiac phenotype induced by PPAR $\alpha$ overexpression mimics that caused by diabetes mellitus. $J$ Clin Invest 109: 121-130, 2002.

22. Frey N, Katus HA, Olson EN, and Hill JA. Hypertrophy of the heart: a new therapeutic target? Circulation 109: 15801589, 2004.

23. Goode N, Hughes K, Woodgett JR, and Parker PJ. Differential regulation of glycogen synthase kinase-3 beta by protein kinase C isotypes. J Biol Chem 267: 16878-16882, 1992.

24. Gopinathan L, Hannon DB, Peters JM, and Heuvel JPV. Regulation of peroxisome proliferator-activated receptor- $\alpha$ by MDM2. Toxicol Sci 108: 48-58, 2009.

25. Gottlieb TM and Oren M. p53 in growth control and neoplasia. Biochim Biophys Acta 1287: 77-102, 1996.

26. Gupta A, Gupta S, Young D, Das B, McMahon J, and Sen S. Impairment of ultrastructure and cytoskeleton during progression of cardiac hypertrophy to heart failure. Lab Invest 90: 520-530, 2010.

27. Haemmerle G, Moustafa T, Woelkart G, Büttner S, Schmidt A, van de Weijer T, Hesselink M, Jaeger D, Kienesberger PC, Zierler K, Schreiber R, Eichmann T, Kolb D, Kotzbeck P, Schweiger M, Kumari M, Eder S, Schoiswohl G, Wongsiriroj N, Pollak NM, Radner FP, Preiss-Landl K, Kolbe T, Rülicke T, Pieske B, Trauner M, Lass A, Zimmermann R, Hoefler G, Cinti S, Kershaw EE, Schrauwen P, Madeo F, Mayer B, and Zechner R. ATGLmediated fat catabolism regulates cardiac mitochondrial function via PPAR-[alpha] and PGC-1. Nat Med 17: 10761085, 2011.

28. Halestrap AP, Clarke SJ, and Javadov SA. Mitochondrial permeability transition pore opening during myocardial reperfusion-a target for cardioprotection. Cardiovasc Res 61: 372-385, 2004.

29. Haupt Y, Rowan S, Shaulian E, Vousden KH, and Oren M. Induction of apoptosis in HeLa cells by trans-activationdeficient p53. Genes Dev 9: 2170-2183, 1995.

30. Juhaszova M, Zorov DB, Kim SH, Pepe S, Fu Q, Fishbein KW, Ziman BD, Wang S, Ytrehus K, Antos CL, Olson EN, and Sollott SJ. Glycogen synthase kinase- $3 \beta$ mediates convergence of protection signaling to inhibit the mitochondrial permeability transition pore. J Clin Invest 113: 1535-1549, 2004.

31. Jürgensmeier JM, Xie Z, Deveraux Q, Ellerby L, Bredesen $\mathrm{D}$, and Reed JC. Bax directly induces release of cytochrome c from isolated mitochondria. Proc Natl Acad Sci USA 95: 4997-5002, 1998.

32. Kolwicz SC, Olson DP, Marney LC, Garcia-Menendez L, Synovec RE, and Tian R. Cardiac-specific deletion of acetyl CoA carboxylase 2 prevents metabolic remodeling during pressure-overload hypertrophy. Circ Res 111: 728$738,2012$.

33. Koopman WJ, Visch HJ, Smeitink JA, and Willems PH. Simultaneous quantitative measurement and automated analysis of mitochondrial morphology, mass, potential, and 
motility in living human skin fibroblasts. Cytometry A 69: 1-12, 2006.

34. Kubbutat MH, Ludwig RL, Ashcroft M, and Vousden KH. Regulation of Mdm2-directed degradation by the C terminus of p53. Mol Cell Biol 18: 5690-5698, 1998.

35. Kwong JQ and Molkentin JD. Physiological and pathological roles of the mitochondrial permeability transition pore in the heart. Cell Metab 21: 206-214, 2015.

36. Leone TC, Weinheimer CJ, and Kelly DP. A critical role for the peroxisome proliferator-activated receptor $\alpha$ $(\operatorname{PPAR} \alpha)$ in the cellular fasting response: the $\operatorname{PPAR} \alpha$-null mouse as a model of fatty acid oxidation disorders. Proc Natl Acad Sci USA 96: 7473-7478, 1999.

37. Li M, Luo J, Brooks CL, and Gu W. Acetylation of p53 inhibits its ubiquitination by Mdm2. J Biol Chem 277: 50607-50611, 2002.

38. Li CH, Cheng YW, Liao PL, and Kang JJ. Translocation of p53 to mitochondria is regulated by its lipid binding property to anionic phospholipids and it participates in cell death control. Neoplasia 12: 150-160, 2010.

39. Li J, Qi M, Li C, Shi D, Zhang D, Xie D, Yuan T, Feng J, Liu Y, Liang D, Xu X, Chen J, Xu L, Zhang H, Ye J, Lv F, Huang J, Peng L, and Chen YH. Tom70 serves as a molecular switch to determine pathological cardiac hypertrophy. Cell Res 24: 977-993, 2014.

40. Marouco D, Garabadgiu AV, Melino G, and Barlev NA. Lysine-specific modifications of p53: a matter of life and death? Oncotarget 4: 1556-1571, 2013.

41. Mathur A, Hong Y, Kemp BK, Barrientos AA, and Erusalimsky JD. Evaluation of fluorescent dyes for the detection of mitochondrial membrane potential changes in cultured cardiomyocytes. Cardiovasc Res 46: 126-138, 2000.

42. Maurer U, Charvet C, Wagman AS, Dejardin E, and Green DR. Glycogen synthase kinase-3 regulates mitochondrial outer membrane permeabilization and apoptosis by destabilization of MCL-1. Mol Cell 21: 749-760, 2006.

43. Mir SA, Chatterjee A, Mitra A, Pathak K, Mahata SK, and Sarkar S. Inhibition of signal transducer and activator of transcription 3 (STAT3) attenuates interleukin-6 (IL-6)induced collagen synthesis and resultant hypertrophy in rat heart. J Biol Chem 287: 2666-2677, 2012.

44. Mitra A, Basak T, Datta K, Naskar S, Sengupta S, and Sarkar S. Role of $\alpha$-crystallin B as a regulatory switch in modulating cardiomyocyte apoptosis by mitochondria or endoplasmic reticulum during cardiac hypertrophy and myocardial infarction. Cell Death Dis 4: e582, 2013.

45. Mitra A, Ray A, Datta R, Sengupta S, and Sarkar S. Cardioprotective role of P38 MAPK during myocardial infarction via parallel activation of $\alpha$-crystallin B and Nrf2. $J$ Cell Physiol 229: 1272-1282, 2014.

46. Mitra A, Basak T, Ahmad S, Datta K, Datta R, Sengupta S, and Sarkar S. Comparative proteome profiling during cardiac hypertrophy and myocardial infarction reveals altered glucose oxidation by differential activation of pyruvate dehydrogenase E1 component subunit $\beta$. J Mol Biol 427: 2104-2120, 2015.

47. Mitra A, Datta R, Rana S, and Sarkar S. Modulation of NFKB1/p50 by ROS leads to impaired ATP production during MI compared to cardiac hypertrophy. J Cell Biochem 119: 1575-1590, 2018.

48. Mitsuishi M, Miyashita K, Muraki A, and Itoh H. Angiotensin II reduces mitochondrial content in skeletal muscle and affects glycemic control. Diabetes 58: 710717, 2009.
49. Momand $\mathrm{J}, \mathrm{Wu} \mathrm{HH}$, and Dasgupta G. MDM2-master regulator of the p53 tumor suppressor protein. Gene 242: 15-29, 2000.

50. Nemajerova A, Erster S, and Moll UM. The posttranslational phosphorylation and acetylation modification profile is not the determining factor in targeting endogenous stress-induced p53 to mitochondria. Cell Death Differ 12: 197-200, 2005.

51. Neubauer S. The failing heart-an engine out of fuel. $N$ Engl J Med 356: 1140-1151, 2007.

52. Ngok-Ngam $P$, Watcharasit $P$, Thiantanawat A, and Satayavivad J. Pharmacological inhibition of GSK3 attenuates DNA damage-induced apoptosis via reduction of p53 mitochondrial translocation and Bax oligomerization in neuroblastoma SH-SY5Y cells. Cell Mol Biol Lett 18: 58-74, 2013.

53. Oliner JD, Pietenpol JA, Thiagalingam S, Gyuris J, Kinzler $\mathrm{KW}$, and Vogelstein B. Oncoprotein MDM2 conceals the activation domain of tumour suppressor p53. Nature 362: 857-860, 1993.

54. Park SY, Cho YR, Finck BN, Kim HJ, Higashimori T, Hong EG, Lee MK, Danton C, Deshmukh S, Cline GW, Wu JJ, Bennett AM, Rothermel B, Kalinowski A, Russell KS, Kim YB, Kelly DP, and Kim JK. Cardiac-specific overexpression of peroxisome proliferator-activated receptor- $\alpha$ causes insulin resistance in heart and liver. Diabetes 54: 2514-2524, 2005.

55. Picard M, White K, and Turnbull DM. Mitochondrial morphology, topology, and membrane interactions in skeletal muscle: a quantitative three-dimensional electron microscopy study. J Appl Physiol (1985) 114: 161-171, 2013.

56. Poyurovsky MV, Katz C, Laptenko O, Beckerman R, Lokshin M, Ahn J, Byeon IJ, Gabizon R, Mattia M, Zupnick A, Brown LM, Friedler A, and Prives C. The $\mathrm{C}$ terminus of p53 binds the N-terminal domain of MDM2. Nat Struct Mol Biol 17: 982-989, 2010.

57. Rana S, Datta K, Reddy TL, Chatterjee E, Sen P, PalBhadra M, Bhadra U, Pramanik A, Pramanik P, ChawlaSarkar M, and Sarkar S. A spatio-temporal cardiomyocyte targeted vector system for efficient delivery of therapeutic payloads to regress cardiac hypertrophy abating bystander effect. J Control Release 200: 167-178, 2015.

58. Ray A, Rana S, Banerjee D, Mitra A, Datta R, Naskar S, and Sarkar S. Improved bioavailability of targeted curcumin delivery efficiently regressed cardiac hypertrophy by modulating apoptotic load within cardiac microenvironment. Toxicol Appl Pharmacol 290: 54-65, 2016.

59. Rosca MG, Vazquez EJ, Kerner J, Parland W, Chandler MP, Stanley W, Sabbah HN, and Hoppel CL. Cardiac mitochondria in heart failure: decrease in respirasomes and oxidative phosphorylation. Cardiovasc Res 80: 30-39, 2008.

60. Sack MN, Rader TA, Park S, Bastin J, McCune SA, and Kelly DP. Fatty acid oxidation enzyme gene expression is downregulated in the failing heart. Circulation 94: 28372842, 1996.

61. Sadoshima J and Izumo S. The cellular and molecular response of cardiac myocytes to mechanical stress. Annu Rev Physiol 59: 551-571, 1997.

62. Sarkar S, Chawla-Sarkar M, Young D, Nishiyama K, Rayborn ME, Hollyfield JG, and Sen S. Myocardial cell death and regeneration during progression of cardiac hypertrophy to heart failure. J Biol Chem 279: 52630-52642, 2004.

63. Sutherland C, Leighton IA, and Cohen P. Inactivation of glycogen synthase kinase- $3 \beta$ by phosphorylation: new kinase connections in insulin and growth-factor signalling. Biochem J 296: 15-19, 1993. 
64. Wadgaonkar $\mathrm{R}$ and Collins $\mathrm{T}$. Murine double minute (MDM2) blocks p53-coactivator interaction, a new mechanism for inhibition of p53-dependent gene expression. $J$ Biol Chem 274: 13760-13767, 1999.

65. Wang XW, Vermeulen W, Coursen JD, Gibson M, Lupold SE, Forrester K, Xu G, Elmore L, Yeh H, Hoeijmakers JH, and Harris CC. The XPB and XPD DNA helicases are components of the p53-mediated apoptosis pathway. Genes Dev 10: 1219-1232, 1996.

66. Wang W, Fang H, Groom L, Cheng A, Zhang W, Liu J, Wang X, Li K, Han P, Zheng M, Yin J, Wang W, Mattson MP, Kao JP, Lakatta EG, Sheu SS, Ouyang K, Chen J, Dirksen RT, and Cheng H. Superoxide flashes in single mitochondria. Cell 134: 279-290, 2008.

67. Watcharasit P, Bijur GN, Zmijewski JW, Song L, Zmijewska A, Chen X, Johnson GV, and Jope RS. Direct, activating interaction between glycogen synthase kinase-3 $\beta$ and p53 after DNA damage. Proc Natl Acad Sci USA 99: 7951-7955, 2002.

68. Watcharasit P, Bijur GN, Song L, Zhu J, Chen X, and Jope RS. Glycogen synthase kinase- $3 \beta$ (GSK3 $\beta$ ) binds to and promotes the actions of p53. J Biol Chem 278: 4887248879, 2003.

69. Zielonka J, Vasquez-Vivar J, and Kalyanaraman B. Detection of 2-hydroxyethidium in cellular systems: a unique marker product of superoxide and hydroethidine. Nat Protoc 3: 8-21, 2008.

70. Zhang L, Jaswal JS, Ussher JR, Sankaralingam S, Wagg C, Zaugg M, and Lopaschuk GD. Cardiac insulin-resistance and decreased mitochondrial energy production precede the development of systolic heart failure after pressureoverload hypertrophy. Circ Heart Fail 6: 1039-1048, 2013.

Address correspondence to:
Dr. Sagartirtha Sarkar
Department of Zoology
University of Calcutta
35, Ballygunge Circular Road
Kolkata 700019
West Bengal
India

E-mail: sagartirtha.sarkar@gmail.com

Date of first submission to ARS Central, October 3, 2017; date of final revised submission, March 7, 2018; date of acceptance, March 31, 2018.

$\begin{aligned} & \quad \text { Abbreviations Used } \\ & \beta-\mathrm{MHC}=\beta \text {-myosin heavy chain } \\ & \Delta \Psi \mathrm{m}=\text { mitochondrial membrane potential } \\ & \mathrm{aa}=\text { amino acid } \\ & \mathrm{ANF}=\text { atrial natriuretic factor } \\ & \mathrm{AngII}=\text { angiotensin } \mathrm{II} \\ & \mathrm{AR}=\text { aspect ratio }\end{aligned}$

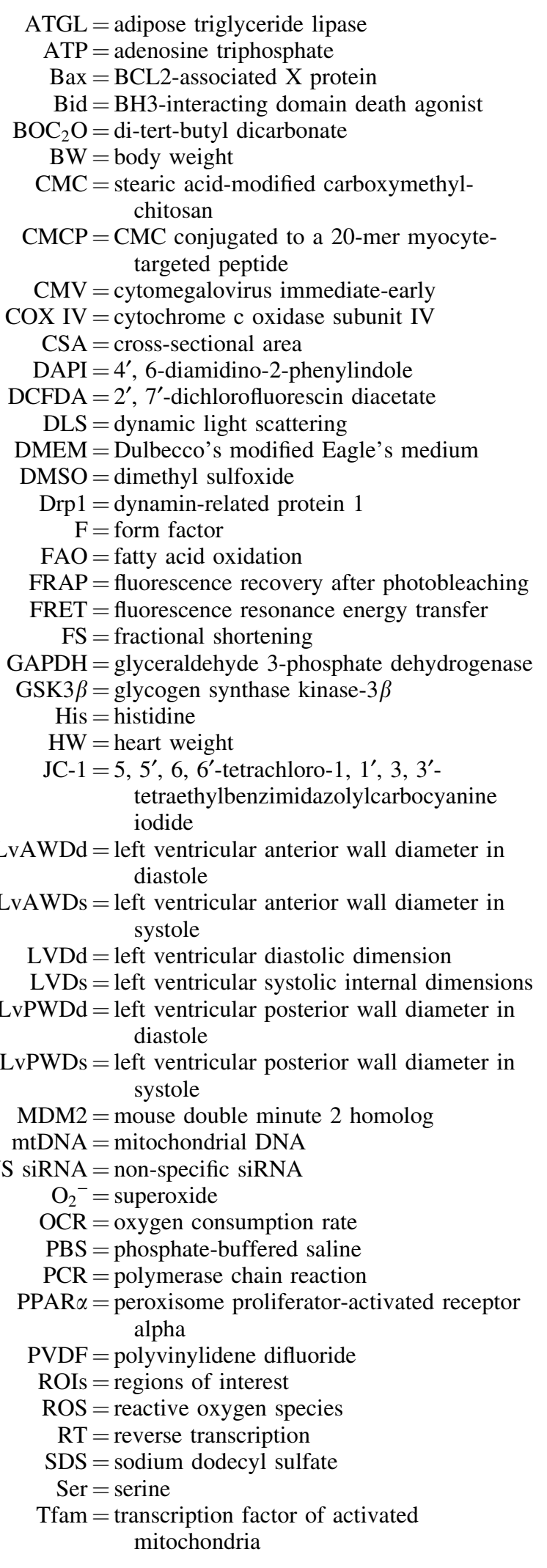

